\title{
ISOCAM view of the starburst galaxies M 82, NGC 253 and NGC 1808*
}

\author{
N. M. Förster Schreiber ${ }^{1, \star \star}$, M. Sauvage ${ }^{1}$, V. Charmandaris ${ }^{2,3}$, O. Laurent ${ }^{1,4}$, P. Gallais ${ }^{1}$, \\ I. F. Mirabel ${ }^{1,5}$, and L. Vigroux ${ }^{1}$ \\ ${ }^{1}$ CEA/DSM/DAPNIA/Service d'Astrophysique, CE Saclay, 91191 Gif-sur-Yvette Cedex, France \\ 2 Astronomy Department, Cornell University, Ithaca, NY 14853, USA \\ 3 Observatoire de Paris ${ }^{\star \star \star}$, LERMA, 61 avenue de l'Observatoire, 75014 Paris, France \\ ${ }^{4}$ Max-Planck-Institute für extraterrestrische Physik, Postfach 1312, 85741 Garching, Germany \\ 5 Instituto de Astronomía y Física del Espacio, cc 67, suc 28, 1428 Buenos Aires, Argentina
}

Received 22 August 2002 / Accepted 15 November 2002

\begin{abstract}
We present results of mid-infrared $\lambda=5.0-16.5 \mu \mathrm{m}$ spectrophotometric imaging of the starburst galaxies M 82, NGC 253, and NGC 1808 from the ISOCAM instrument on board the Infrared Space Observatory. The mid-infrared spectra of the three galaxies are very similar in terms of features present. The $\lambda \gtrsim 11 \mu \mathrm{m}$ continuum attributed to very small dust grains (VSGs) exhibits a large spread in intensity relative to the short-wavelength emission. We find that the $15 \mu \mathrm{m}$ dust continuum flux density correlates well with the fine-structure [ $\mathrm{Ar}$ II] $6.99 \mu \mathrm{m}$ line flux and thus provides a good quantitative indicator of the level of star formation activity. By contrast, the $\lambda=5-11 \mu \mathrm{m}$ region dominated by emission from polycyclic aromatic hydrocarbons (PAHs) has a nearly invariant shape. Variations in the relative intensities of the PAH features are nevertheless observed, at the $20 \%-100 \%$ level. We illustrate extinction effects on the shape of the mid-infrared spectrum of obscured starbursts, emphasizing the differences depending on the applicable extinction law and the consequences for the interpretation of PAH ratios and extinction estimates. The relative spatial distributions of the PAH, VSG, and [Ar II] $6.99 \mu \mathrm{m}$ emission between the three galaxies exhibit remarkable differences. The $\lesssim 1 \mathrm{kpc}$ size of the mid-infrared source is much smaller than the optical extent of our sample galaxies and $70 \%-100 \%$ of the IRAS $12 \mu \mathrm{m}$ flux is recovered within the ISOCAM $\leq 1.5 \mathrm{arcmin}^{2}$ field of view, indicating that the nuclear starburst dominates the total mid-infrared emission while diffuse light from quiescent disk star formation contributes little.
\end{abstract}

Key words. galaxies: individual: M 82, NGC 253, NGC 1808 - galaxies: ISM - galaxies: starburst - infrared: galaxies infrared: ISM

\section{Introduction}

As hosts of vigorous episodes of star formation activity, starburst galaxies in the local Universe offer a unique opportunity to study the star formation process in extreme environments reminiscent of the conditions presumably prevailing in primordial galaxies. Studies in recent years have underscored the prominent role of starbursts in galaxy formation and evolution as well as their significant contribution to the

Send offprint requests to: N. M. Förster Schreiber,

e-mail: forster@strw.leidenuniv.nl

* Based on observations with $I S O$, an ESA project with instruments funded by ESA member states (especially the PI countries: France, Germany, The Netherlands, and the UK), and with participation of ISAS and NASA.

$\star \star$ Present address: Leiden Observatory, PO Box 9513, 2300 RA Leiden, The Netherlands.

$\star \star \star$ Chercheur Associé. extragalactic background, and to the chemical enrichment and heating of the intergalactic medium up to the highest redshifts (e.g. Steidel et al. 1996; Puget et al. 1996; Smail et al. 1997; Franx et al. 1997; Pettini et al. 1998; Elbaz et al. 2002). Understanding the starburst phenomenon is thus a key issue of local and cosmological relevance.

Nuclear starburst galaxies generally emit the bulk of their luminosity in the infrared, primarily as reprocessed radiation by interstellar dust grains heated by important populations of hot massive stars. In addition, a substantial fraction - if not most - of the star formation in starburst systems is heavily obscured at optical and ultraviolet wavelengths by large amounts of dust. This has been particularly dramatically illustrated by mid-infrared imaging of the colliding pair NGC 4038/4039 (Mirabel et al. 1998). Studies at infrared wavelengths are thus crucial in defining the properties of starburst galaxies and the interplay between the primary energy sources and the reprocessing material. The growing evidence for the presence and 
significant role of dust in distant galaxies further emphasizes the need for infrared investigations of nearby dust-rich templates (e.g. Rowan-Robinson 2001; Blain et al. 2002; and references therein). However, except for a limited sample, the detailed spatial and spectral energy distributions of starburst galaxies in this regime are still poorly known. Progress has been hindered by instrumental limitations, and by the difficulties inherent to ground-based and air-borne observations at these wavelengths.

The Infrared Space Observatory (ISO; Kessler et al. 1996) has revolutionized the field of infrared astronomy (see the reviews by Cesarsky \& Sauvage 1999; Genzel \& Cesarsky 2000), with unprecedented capabilities compared to its predecessor the Infrared Astronomical Satellite (IRAS). In the mid-infrared (MIR, $\lambda=5.0-16.5 \mu \mathrm{m}$ ), the camera ISOCAM (Cesarsky et al. 1996c) provided an improvement in sensitivity and spatial resolution by factors of $\sim 1000$ and $\sim 60$, respectively. In addition to broad- and narrow-band filters, ISOCAM featured continuously variable filters (CVFs) allowing arcsecond-scale spectrophotometric imaging with full wavelength coverage at a resolution of $R \equiv \lambda / \Delta \lambda \sim 40$. The MIR regime contains a variety of key features tracing different components of the interstellar medium (ISM) and is subject to relatively little extinction by dust $\left(A_{5-17 \mu \mathrm{m}}<0.1 A_{V}\right)$. Spectrophotometric observations permit studies of the most prominent features to characterize the spatial distribution and infer the properties of the ISM and exciting sources, deep into obscured star-forming regions.

In this paper, we present results of ISOCAM CVF observations of M 82, NGC 253, and NGC 1808. M 82 and NGC 253 are two of the nearest and brightest starburst galaxies (at distances of $D=3.3$ and $2.5 \mathrm{Mpc}$, respectively, $1^{\prime \prime}=16$ and 12 pc; Freedman \& Madore 1988; Davidge \& Pritchet 1990). Both have been extensively studied in all accessible spectral regions and have often been considered as prototypical objects for the starburst phenomenon (see Telesco 1988; Rieke et al. 1993; and Engelbracht et al. 1998 for reviews). NGC 1808 is a more distant system $\left(D=10.9 \mathrm{Mpc}\right.$ for $H_{0}=$ $75 \mathrm{~km} \mathrm{~s}^{-1} \mathrm{Mpc}^{-1}, 1^{\prime \prime}=53 \mathrm{pc}$; Sandage \& Tammann 1987) which closely resembles M 82 and NGC 253 in its global properties (e.g. Dahlem et al. 1990; Junkes et al. 1995). In all three galaxies, starburst activity takes place within the central $\sim 0.1-$ $1 \mathrm{kpc}$ regions which are severely obscured at optical and ultraviolet wavelengths. The large extinction levels likely result in part from high disk inclination angles of $80^{\circ}, 78^{\circ}$, and $57^{\circ}$ for M 82, NGC 253, and NGC 1808 (Götz et al. 1990; Pence 1981; Reif et al. 1982).

At comparable infrared luminosities ${ }^{1} L_{\mathrm{IR}} \equiv L_{8-1000 \mu \mathrm{m}}$ of $3.7 \times 10^{10}, 1.4 \times 10^{10}$, and $3.4 \times 10^{10} L_{\odot}$ for M 82, NGC 253 , and NGC 1808, these galaxies also share key morphological features including a kiloparsec-scale stellar bar, large amounts of molecular gas in the central $\sim 1 \mathrm{kpc}$ predominantly concentrated in circumnuclear ring-, spiral-, or bar-like structures, populations of compact and luminous "super star clusters" as well as of compact non-thermal radio sources associated with supernova remnants, and a large-scale outflowing starburst

\footnotetext{
${ }^{1}$ As computed from the IRAS fluxes following the prescription of Sanders \& Mirabel (1996).
}

wind (Förster Schreiber et al. 2001; Engelbracht et al. 1998; Tacconi-Garman et al. 1996; and references therein). The triggering of starburst activity in M 82 is generally attributed to gravitational interaction as evidenced by the extended filamentary tidal features in $\mathrm{H}_{\mathrm{I}}$ gas distribution threading M 82 and its neighbours M 81 and NGC 3077 or, alternatively, to the stellar bar which may itself have been induced by the interaction (e.g. Yun et al. 1993, 1994). In NGC 253, the bar itself appears to be the primary mechanism responsible for the onset of starburst activity as there are no obvious signatures of interaction with a nearby companion, although a past merger or accretion event involving a small galaxy has been suggested based on kinematical evidence (e.g. Anantharamaiah \& Goss 1996; Böker et al. 1998). Tidal interaction with NGC 1792 has been proposed for NGC 1808 based on circumstantial evidence (e.g. Dahlem et al. 1990; Koribalski et al. 1993). The recent high sensitivity H I observations by Dahlem et al. (2001) do not indicate the presence of any tidal feature, dwarf galaxy or other debris that could support a close passage in the past, leaving the stellar bar as more probable trigger or a possible merger/accretion event.

In view of their similar nature, we focus on the comparison of the spatial and spectral properties of M 82, NGC 253, and NGC 1808 at MIR wavelengths. Though small, this sample also allows us to probe the transition regime between normal spiral and irregular galaxies and the more extreme luminous and ultraluminous infrared galaxies (LIRGs and ULIRGs for which $L_{\mathrm{IR}}>10^{11}$ and $>10^{12} L_{\odot}$, respectively). Section 2 describes the observations and data reduction procedure, and Sect. 3 presents the results. Section 4 discusses the origin and spatial distibution of the continuum and emission features. Section 5 addresses the issues of extinction effects, variations in spectral properties, and indicators of star formation activity. Section 6 summarizes the paper.

\section{Observations and data reduction}

We observed M 82, NGC 253, and NGC 1808 with ISOCAM (Cesarsky et al. 1996c) on board ISO (Kessler et al. 1996) as part of the guaranteed time program CAMACTIV (P. I. I. F. Mirabel). We used the CVFs to cover the entire $\lambda=5.0$ $16.5 \mu \mathrm{m}$ range with a resolution of $R \equiv \lambda / \Delta \lambda \approx 35$ to 45 and increments $\delta \lambda=0.06$ to $0.1 \mu \mathrm{m}$ from short to long wavelengths. For all three sources, twelve single-frame exposures of $2.1 \mathrm{~s}$ were recorded per wavelength channel, for total integration times of about $1^{\mathrm{h}} 05^{\mathrm{m}}$. We selected the $3^{\prime \prime}$ pixel ${ }^{-1}$ scale for M 82 and NGC 1808, and the $1.5^{\prime \prime}$ pixel $^{-1}$ scale for NGC 253; total fields of view of $96^{\prime \prime} \times 96^{\prime \prime}$ and $48^{\prime \prime} \times 48^{\prime \prime}$ are thus covered by the $32 \times 32$ pixels detector array. Table 1 presents the details of the observations. The areas observed with ISOCAM cover almost entirely the MIR sources in each galaxy (Sect. 4) and are indicated on optical images in Fig. 1. This emphasizes the small MIR source sizes compared to the optical extent of the galaxies and that most of the MIR emission originates in their nuclear regions. 

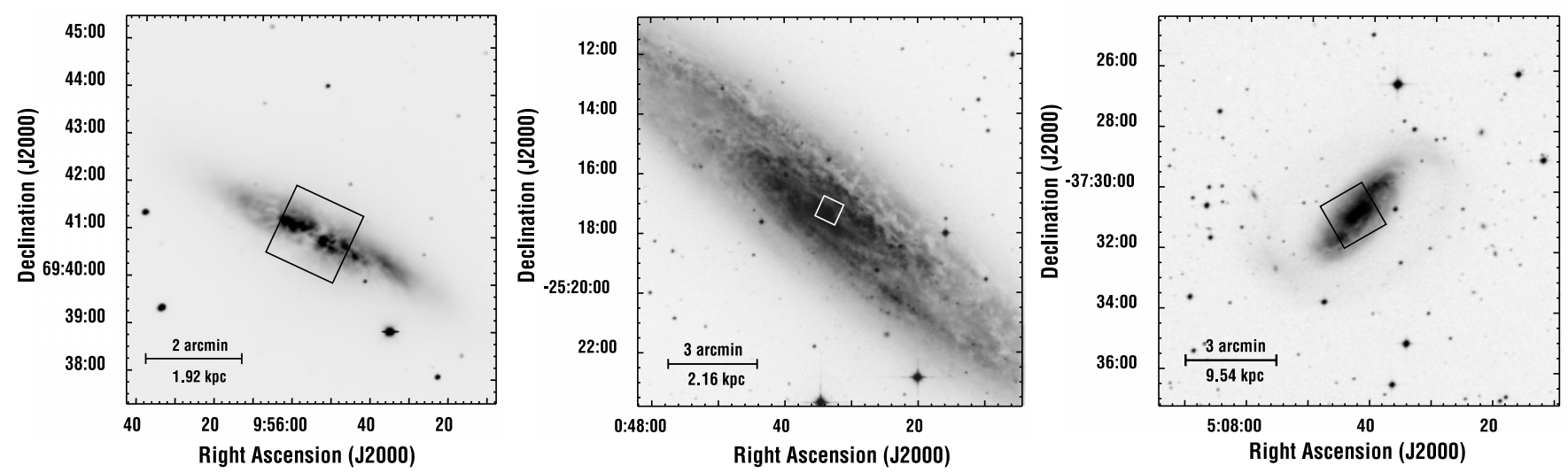

Fig. 1. The ISOCAM CVF field of view, indicated by a square, is superimposed on publicly available images in the $V$ band for M 82 (left), and $R$ band for NGC 253 and NGC 1808 (middle and right).

Table 1. Log of the observations.

\begin{tabular}{llllllll}
\hline \hline Source & Date & Revolution & $\begin{array}{l}\alpha_{2000} \\
\left({ }^{\mathrm{h}}:{ }^{\mathrm{m}}:{ }^{\mathrm{s}}\right)\end{array}$ & $\begin{array}{l}\delta_{2000} \\
\left({ }^{\circ}:^{\prime}:{ }^{\prime \prime}\right)\end{array}$ & $\begin{array}{l}\text { PA }^{a} \\
(\text { degree })\end{array}$ & $\begin{array}{l}\text { Pixel scale } \\
(\operatorname{arcsec})\end{array}$ & $\begin{array}{c}t_{\text {int }}{ }^{b} \\
(\mathrm{~s})\end{array}$ \\
\hline M 82 & 1996 Mar 19 & 123 & $09: 55: 51.8$ & $+69: 40: 45.8$ & 212 & 3.0 & 25.2 \\
NGC 253 & 1997 Jan 03 & 414 & $00: 47: 32.9$ & $-25: 17: 18.3$ & 290 & 1.5 & 25.2 \\
NGC 1808 & 1997 Oct 12 & 697 & $05: 07: 42.3$ & $-37: 30: 46.1$ & 64 & 3.0 & 25.2 \\
\hline
\end{tabular}

${ }^{a}$ Position angle of the vertical axis of the ISOCAM array relative to the north and increasing counterclockwise.
${ }^{b}$ Total integration time per wavelength channel.

We reduced the data using the CAM Interactive Analysis package (CIA) ${ }^{2}$ following the procedures described in the ISOCAM User's Manual (Delaney 1997). We first subtracted the dark current using a model of the secular evolution of ISOCAM's dark current (Biviano et al. 1997), removed cosmic ray hits by applying a multiresolution median filtering technique (Starck et al. 1999), and corrected for transient detector memory effects using the "Fouks-Schubert" algorithm (Coulais $\&$ Abergel 2000). We then combined the individual exposures for each wavelength channel with the help of a shift-and-add algorithm accounting for the satellite jitter motions (typical amplitude of $\approx 1^{\prime \prime}$ ) and performed absolute flux calibration based on the sensitivity calibration files provided in CIA version 3.0.

For proper comparison of fluxes and maps obtained at different wavelengths, we smoothed the CVF channels to a common angular resolution and registered them appropriately. The point spread function (PSF) varies throughout the CVF scans, with theoretical full-width at half-maximum $F W H M=3.1^{\prime \prime}-$ 5.6" for the $3^{\prime \prime}$ pixel $^{-1}$ scale and 2.0" $-5.2^{\prime \prime}$ for the $1.5^{\prime \prime}$ pixel $^{-1}$ scale, over the range 5.5-16.5 $\mu \mathrm{m}$. We convolved the data to a final resolution of $5.6^{\prime \prime}$ for M 82 and NGC 1808, and $5.2^{\prime \prime}$ for NGC 253, corresponding to a linear resolution of 90,297 , and 62 pc, respectively. We computed the transfer functions (close to Gaussians in profile) using the library of theoretical PSFs for ISOCAM and a CLEAN algorithm.

${ }^{2}$ CIA is a joint development by the ESA Astrophysics Division and the ISOCAM consortium led by the ISOCAM P.I. C. Cesarsky, Direction des Sciences de la Matière, C.E.A. Saclay, France.
The small-scale structure is thus smeared out in the higher resolution channels but no important spatial feature is lost.

The source position varies slightly with wavelength due to telescope motions and changes in optical path, with the largest offset occurring near $9.2 \mu \mathrm{m}$ between the short and long wavelength segments of the CVFs. Since no unresolved point source is detected in the data, we determined the position offsets from cross-correlation over the entire field of view, taking as references images integrated over various wavelength intervals. Effects due to the different spatial distributions of emission features were easily identified and ignored in the determination of the instrumental shifts. We fitted straight lines to the crosscorrelation offsets for each CVF segment. For the $3^{\prime \prime}$ pixel $^{-1}$ scale, the horizontal, or $x$-axis offsets relative to $9.2 \mu \mathrm{m}$ in the short and long wavelength segments did not exceed 0.3 pixel and 0.5 pixel, respectively, with a jump of 0.7 pixel at the transition wavelength. Shifts along the $y$-axis were negligible throughout the entire wavelength range $(<0.2$ pixel). For the $1.5^{\prime \prime}$ pixel $^{-1}$ scale, both $x$ - and $y$-axis offsets were $\leq 0.3$ pixel within each CVF segment, with jumps at $9.2 \mu \mathrm{m}$ of 0.2 pixel only.

We did not attempt to correct for instrumental flat-field, straylight, and ghost images generated by multiple reflections between the detector and filters since appropriate calibration files for the CVF mode were not available. Based on theoretical models for point-like and extended sources as well as observations of stars and of the zodiacal light (Biviano et al. 1998a,b; Okumura 2000), the combined flat-field, straylight, and ghost 
effects integrated over the entire detector array vary between $\approx 35 \%$ and $10 \%$ of the source flux from 5 to $16 \mu \mathrm{m}$, and exhibit qualitatively similar spatial structures at different wavelengths. The rms noise estimated from existing flat-field images obtained with ISOCAM's broad-band filters is $\sim 10 \%$ for both pixel scales (Biviano et al. 1998b).

Based on available calibration accuracy reports (Blommaert \& Cesarsky 1998 and references therein), the systematic errors of the data processing and flux calibration described above sum up to total uncertainties on the absolute photometry of $\approx 50 \%$. For our analysis, we will consider formal effective uncertainties on the relative fluxes estimated as follows. We measured the dispersion around the mean flux among the individual reduced exposures for each wavelength channel at each detector pixel, included an additional 10\% rms noise for intrinsic pixel-to-pixel variations in sensitivity, and accounted for uncertainties in relative spectral calibration. For M 82 and NGC 253, the latter were evaluated from the comparison with data from the ISO Short Wavelength Spectrometer (SWS; de Graauw et al. 1996) in Sect. 3, amounting to 5\% and $11 \%$, respectively. For NGC 1808, very low flux levels and high noise around $10 \mu \mathrm{m}$, below $6 \mu \mathrm{m}$, and outside of the main emission region led to poor correction for transient memory effects because of the difficulty in determining the initial and stabilized fluxes and of the bad behaviour of the algorithm wherever fluxes become negative. In this case, we estimated the errors in relative spectral calibration by comparing results when applying the transient correction to the data as they were and to the data where all values lower than 0.01 were set to 0.01 , adding a conservative $10 \%$. The median and average differences are of $16 \%$ and a factor of 2. Experimentation showed that the choice of the threshold is of little consequence as long as it is sufficiently smaller than the signal where the transient correction is satisfactory.

Overall, the formal effective uncertainties per pixel and wavelength channel within the brighter emission regions of interest (defined by the "starburst cores" in Sect. 3) have median values of $18 \%, 29 \%$, and $45 \%$ for M 82, NGC 253, and NGC 1808. Computed over the entire ISOCAM field of view, the medians increase to $32 \%, 88 \%$, and a factor of 2.6 as a result of the higher noise towards the edges of the array where little flux is detected.

\section{Results}

\subsection{Mid-infrared spectra and feature identification}

We extracted from the reduced ISOCAM data cubes spectra integrated over various regions: a "starburst core" centered on each galaxy's nucleus ${ }^{3}$ and covering most of the emission source, and a smaller region at the position of the peak observed for the integrated MIR emission. For M 82 and NGC 253, we further included a "disk" corresponding to an annulus outside of the starburst core and the ISOCAM field of view. Due to the higher noise level in the NGC 1808 data, only the starburst core and MIR peak spectra are of sufficient quality for analysis.

\footnotetext{
${ }^{3}$ As defined by the peak of the near-infrared $2 \mu \mathrm{m}$ emission; see Table 2 for coordinates and references.
}
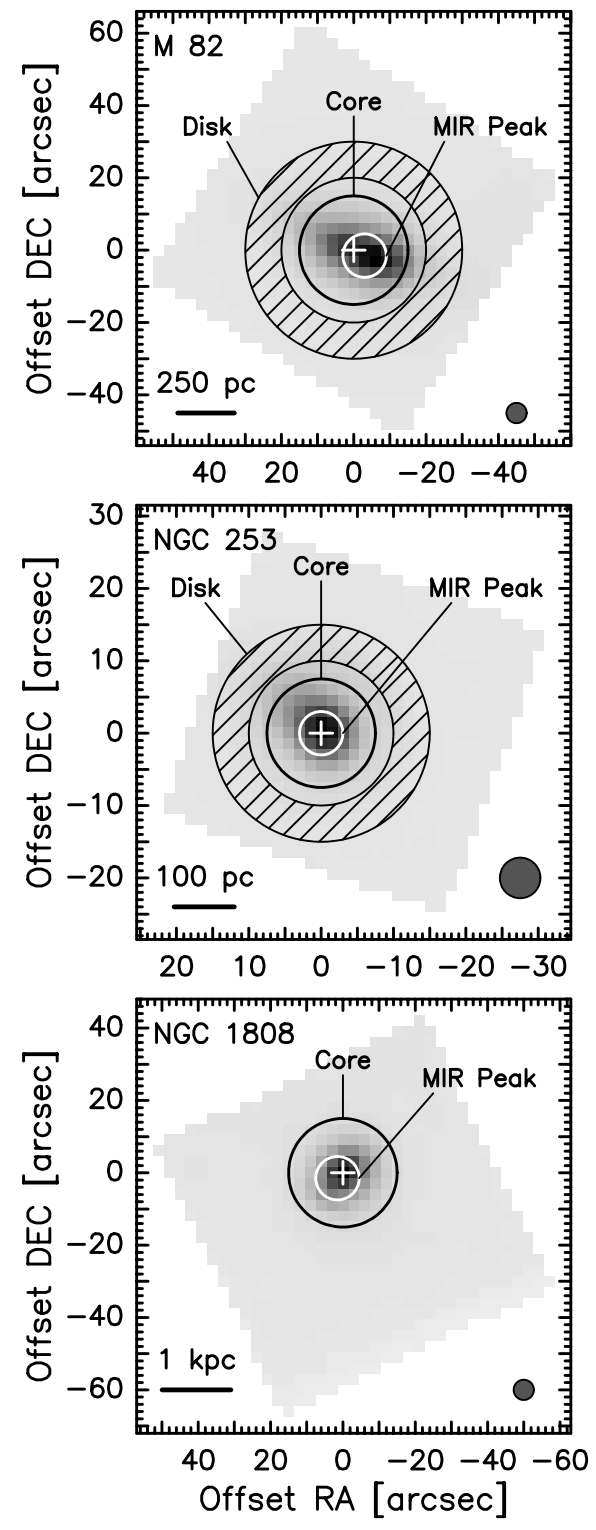

Fig. 2. Selected regions in M 82 (top), NGC 253 (middle), and NGC 1808 (bottom), shown on $\lambda=14.8-15.2 \mu \mathrm{m}$ continuum maps. The starburst core and MIR peak regions are enclosed within the black and white circles, respectively. The disk regions for M 82 and NGC 253 correspond to the hatched areas. The parameters for all regions are given in Table 2. The horizontal bar and the filled circle at the bottom of each panel indicate the physical linear scale and the FWHM of the PSF.

The disk, core, and MIR peak regions were chosen to sample relatively quiescent to intense star-forming activity as traced by e.g. the $15 \mu \mathrm{m}$ continuum emission (see Sects. 3.2 and 5.2). The starburst cores correspond to the apertures generally used in previous studies when referring to the global properties of the starburst in each galaxy.

Figure 2 indicates the selected regions on maps of the $14.8-$ $15.2 \mu \mathrm{m}$ emission. Figure 3 shows the spectra, all normalized to a total $6.0-6.6 \mu \mathrm{m}$ flux density of unity to facilitate comparison of the relative strength of the emission features at $\lambda \lesssim 11 \mu \mathrm{m}$ and of the continuum intensity at longer wavelengths. The formal effective uncertainties are plotted along 
Table 2. Synthetic apertures for selected regions.

\begin{tabular}{lllcclll}
\hline \hline Source & Region & Position & $\begin{array}{l}\Delta \alpha^{a} \\
(\operatorname{arcsec})\end{array}$ & $\begin{array}{l}\Delta \delta^{a} \\
(\operatorname{arcsec})\end{array}$ & $\begin{array}{l}\text { Aperture } \\
(\operatorname{arcsec})\end{array}$ & $\begin{array}{l}f_{6.0-6.6 \mu \mathrm{m}}{ }^{b} \\
(\mathrm{Jy})\end{array}$ & $\begin{array}{l}\text { Uncertainties }^{c} \\
(\%)\end{array}$ \\
\hline M 82 & CAM FOV & $\ldots$ & 0.0 & +5.0 & $81 \times 81$ at PA of $212^{\circ}$ & $45.0 \pm 0.3$ & $6(1)$ \\
& Disk & Nucleus & 0.0 & 0.0 & $20 \leq r \leq 30$ & $9.37 \pm 0.06$ & $3(2)$ \\
& Core & Nucleus & 0.0 & 0.0 & $r \leq 15$ & $19.5 \pm 0.2$ & $2(2)$ \\
& MIR peak & MIR Peak & -3.0 & -1.5 & $r \leq 6$ & $5.74 \pm 0.10$ & $5(5)$ \\
NGC 253 & CAM FOV & $\ldots$ & -4.0 & +1.0 & $40.5 \times 40.5$ at PA 290 & $8.34 \pm 0.73$ & $20(3)$ \\
& Disk & Nucleus & 0.0 & 0.0 & $10 \leq r \leq 15$ & $1.75 \pm 0.09$ & $11(4)$ \\
& Core & Nucleus & 0.0 & 0.0 & $r \leq 7.5$ & $3.90 \pm 0.05$ & $4(3)$ \\
& MIR peak & MIR Peak & 0.0 & 0.0 & $r \leq 3$ & $1.14 \pm 0.03$ & $8(7)$ \\
NGC 1808 & Core & Nucleus & 0.0 & 0.0 & $r \leq 15$ & $1.77 \pm 0.15$ & $57(4)$ \\
& MIR peak & MIR Peak & +1.5 & -1.5 & $r \leq 6$ & $0.74 \pm 0.02$ & $9(8)$ \\
\hline
\end{tabular}

${ }^{a}$ Right ascension and declination offsets relative to the position of the nucleus of each galaxy. For M 82: $\alpha_{2000}=09^{\mathrm{h}} 55^{\mathrm{m}} 52^{\mathrm{s}} .2, \delta_{2000}=$ $+69^{\circ} 40^{\prime} 46^{\prime \prime} 6$ (Dietz et al. 1986). For NGC 253: $\alpha_{2000}=00^{\mathrm{h}} 47^{\mathrm{m}} 33^{\mathrm{s}} \cdot 1, \delta_{2000}=-25^{\circ} 17^{\prime} 18^{\prime \prime} .3$ (Sams et al. 1994). For NGC 1808: $\alpha_{2000}=$ $05^{\mathrm{h}} 07^{\mathrm{m}} 42^{\mathrm{s}} \cdot 3, \delta_{2000}=-37^{\circ} 30^{\prime} 46^{\prime \prime} 3$ (Collison et al. 1994).

${ }^{b}$ Integrated flux density between 6.0 and $6.6 \mu \mathrm{m}$ used for the normalization of the spectra plotted in Fig. 3. The integration area reported for the ISOCAM field of view of M 82 and NGC 253 excludes the edges which were not illuminated or are highly noisy. Quoted uncertainties are computed from the formal effective uncertainties for individual pixels and wavelength channels (see Sect. 2).

$c$ Average of the formal effective uncertainties per wavelength channel for the integrated spectra (median values are given in parenthesis).

with the spectra. Table 2 gives the parameters for the synthetic apertures, the normalizing fluxes, and the average and median uncertainties for each spectrum. Figure 3 also shows for M 82 and NGC 253 the average spectrum of all individual resolution elements within the ISOCAM field of view, normalized as described above, together with the dispersion around the mean, the full range observed, and the typical (median) uncertainties. The resolution elements for M 82 and NGC 253 correspond to rebinned pixels of size $2 \times 2$ and $4 \times 4$ in original detector pixels, respectively.

All spectra look very similar and exhibit the "classical" characteristics observed towards star-forming regions and galaxies: conspicuous broad emission features (the "unidentified infrared bands" or UIBs), a featureless continuum rising importantly at $\lambda \gtrsim 11 \mu \mathrm{m}$, and an apparent dip near $10 \mu \mathrm{m}$. The UIBs are attributed to a family of particles, the nature of which still is debated, stochastically heated by single ultraviolet photons while the long-wavelength continuum is ascribed to very small dust grains between the transient heating and thermal equilibrium regimes depending on grain properties and radiation field intensity (e.g. Léger et al. 1989; Allamandola et al. 1989; Désert et al. 1990; Duley \& Williams 1991; Tielens et al. 1999; see also reviews by Puget \& Léger 1989; Cesarsky \& Sauvage 1999; Genzel \& Cesarsky 2000). We will hereafter refer to these ISM components as "PAHs," adopting the currently popular model in which the UIB carriers consist of polycyclic aromatic hydrocarbon molecules, and "VSGs."

A number of weaker emission features are also detected in the spectra of Fig. 3. However, their identification is problematic at such low spectral resolution due to possible feature blends. To emphasize this point and secure the identifications, Fig. 4 shows the spectra of M 82 and NGC 253 obtained at $R \sim 500-1000$ with the $I S O$-SWS (from Sturm et al. 2000 and Förster Schreiber et al. 2001). The SWS data are also compared with ISOCAM spectra taken in the same apertures, after convolution to the same spectral resolution. The ISOCAM and SWS data agree very well, confirming the accuracy of the absolute and relative flux calibration for both instruments. For M 82, the differences are $5 \%$ on average (24\% at most). For NGC 253, they are of $11 \%$ on average ( $30 \%$ at most) at $\lambda \geq 5.4 \mu \mathrm{m}$ while they reach a factor of two at shorter wavelengths, probably due to residual transient memory effects.

From this comparison (see also Sturm et al. 2000), the features at $6.2,7.7,8.6$, and $11.3 \mu \mathrm{m}$ are unambiguously identified with PAH emission. The broad feature near $12.7 \mu \mathrm{m}$ clearly results from the blending of the PAH $12.7 \mu \mathrm{m}$ band and of the [Ne II] $12.81 \mu \mathrm{m}$ fine-structure line. The [Ne III] $15.56 \mu \mathrm{m}$ line is also blended with the nearby PAH $15.7 \mu \mathrm{m}$ feature. From the SWS data, and with the continuum and integration bandpasses given below, the PAH $12.7 \mu \mathrm{m}$ accounts for about $50 \%$ of the flux in the $12.7 \mu \mathrm{m}$ blend for both galaxies. The PAH $15.7 \mu \mathrm{m}$ contains about $30 \%$ of the flux in the $15.6 \mu \mathrm{m}$ blend for M 82 (the noisier SWS spectrum of NGC 253 makes an estimate difficult). The least contaminated fine-structure line detected with ISOCAM is [Ar II] $6.99 \mu \mathrm{m}$, with the underlying $\mathrm{PAH}$ at $7.0 \mu \mathrm{m}$ and the $\mathrm{H}_{2} 0-0 S(5)$ rotational line at $6.91 \mu \mathrm{m}$ contributing $\approx 20 \%$ and $5 \%$, respectively, to the blend flux in both galaxies. The weak features at $5.65,13.55$, and $14.25 \mu \mathrm{m}$ are identified with PAH bands; the latter is definitely not due to the high excitation [Ne v] $14.32 \mu \mathrm{m}$ line which is undetected in the SWS data. The final identifications in the ISOCAM spectra are given in Fig. 3. Because of the excellent correspondence between features seen in all three galaxies, the identifications for M 82 and NGC 253 are adopted for NGC 1808 as well.

We performed various continuum and feature measurements on the spectra. Table 3 gives the intervals used in the computations and Tables 4 and 5 report the results. The flux densities in the $6.0-9.0 \mu \mathrm{m}$ and $13.5-15.0 \mu \mathrm{m}$ bands are 

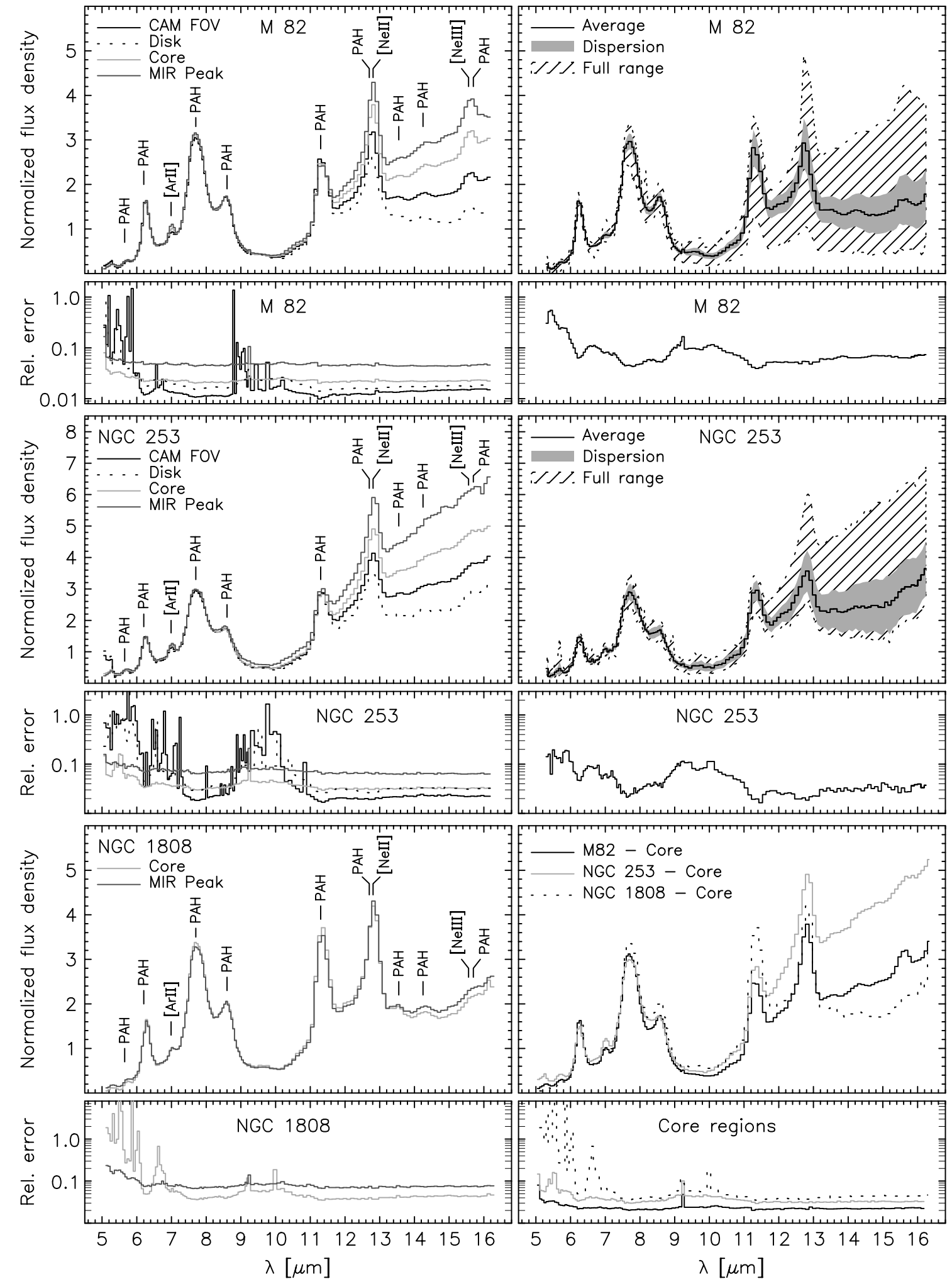

Fig. 3. Mid-infrared spectra of M 82, NGC 253, and NGC 1808. All spectra are normalized to unit total flux density between 6.0 and $6.6 \mu \mathrm{m}$. The three panel pairs to the left show the spectra and relative formal effective uncertainties for selected regions: the ISOCAM field of view, the disk region, the starburst core, and the MIR peak (see labels in each plot); the apertures used, normalizing factors, and typical uncertainties are given in Table 2. The top and middle panel pairs to the right show the average spectrum together with the dispersion, the full range observed, and the median uncertainties derived from all individual resolution elements for M 82 and NGC 253, respectively. The resolution elements correspond to rebinned $2 \times 2$ pixels for M 82 and $4 \times 4$ pixels for NGC 253 (i.e. $6^{\prime \prime} \times 6^{\prime \prime}$ in both cases). The bottom right panel pair compares the spectra of the starburst core regions of each galaxy and their relative uncertainties. The relative uncertainties are expressed as fraction of the measured flux density. 

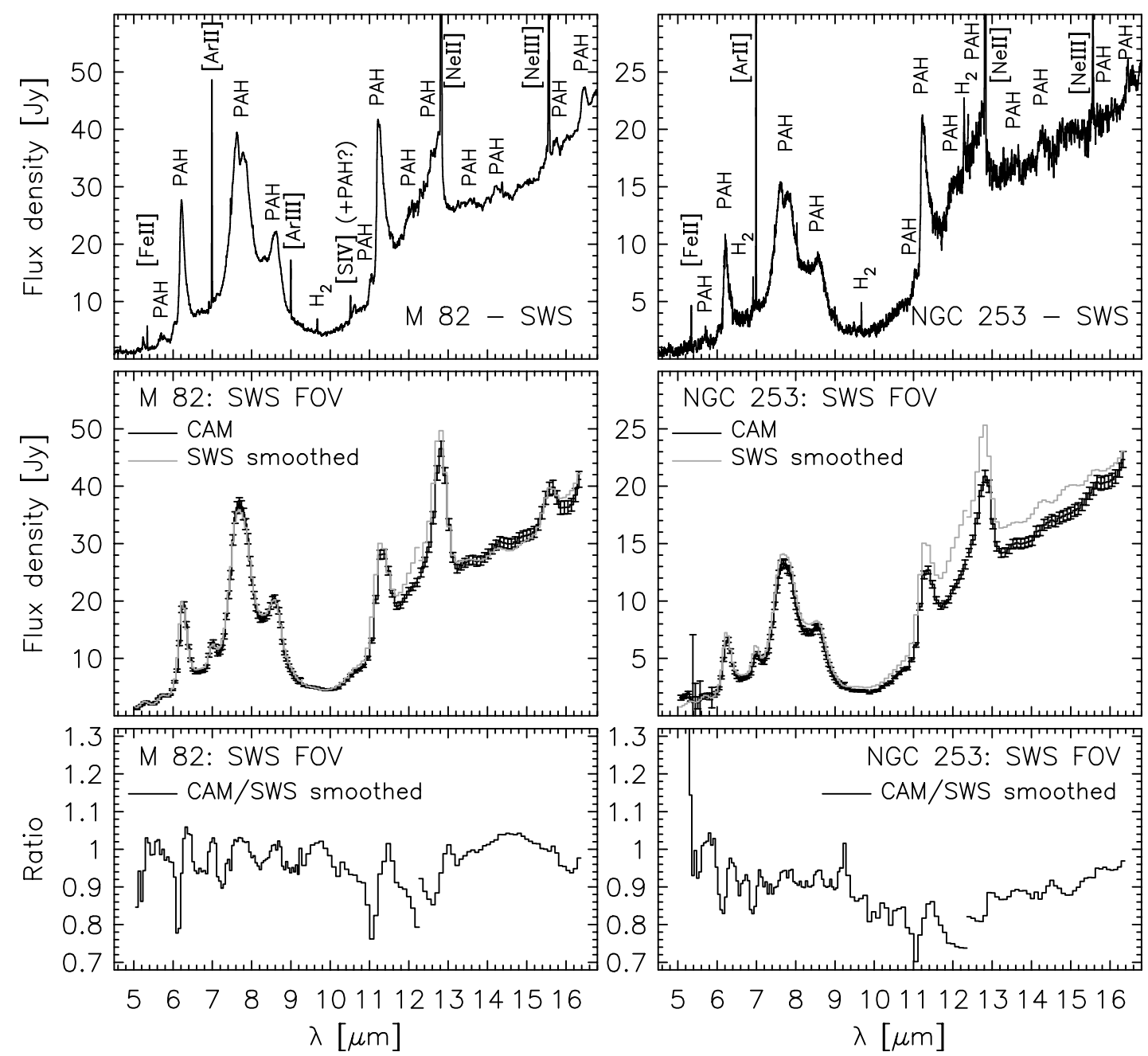

Fig. 4. Comparison of MIR spectra of M 82 and NGC 253 obtained with ISOCAM and SWS. The top panels show the full resolution SWS spectra ( $R \sim 1000$ to 500 from short to long wavelengths), with the identification of the detected emission features (from Förster Schreiber et al. 2001 for M 82, and Sturm et al. 2000 for NGC 253). For M 82, the peak flux densities for the [NeII] $12.81 \mu \mathrm{m}$ and [NeIII] $15.56 \mu \mathrm{m}$ lines are 291.8 Jy and 109.1 Jy. For NGC 253, those for the [ArII] $6.99 \mu \mathrm{m}$, [NeII] $12.81 \mu \mathrm{m}$, and [NeIII] $15.56 \mu \mathrm{m}$ lines are $31.2 \mathrm{Jy}, 140.6 \mathrm{Jy}$, and $35.2 \mathrm{Jy}$. The middle panels compare the SWS data convolved to the resolution of ISOCAM ( $R \sim 35$ to 45 from short to long wavelengths) with the ISOCAM spectra (with uncertainties) integrated over the SWS aperture, while the bottom panels show the ratio of the ISOCAM and smoothed SWS spectra. In the spectral range covered by ISOCAM, the SWS aperture changes from $14^{\prime \prime} \times 20^{\prime \prime}$ shortwards of $\lambda \approx 12.0 \mu \mathrm{m}$ to $14^{\prime \prime} \times 27^{\prime \prime}$ at longer wavelengths. The long wavelength portions of the spectra have been scaled to match the smaller SWS apertures by factors of 0.83 for M 82 and 0.9 for NGC 253, as determined from the continuum level measured in the ISOCAM data.

dominated by emission from PAHs and VSGs, respectively. We chose the narrow continuum bands centered at 5.5 and $15.0 \mu \mathrm{m}$ so as to minimize the contribution from PAHs and other emission lines. In particular, we obtain the same $5.5 \mu \mathrm{m}$ flux densities within $\approx 5 \%$ from the ISOCAM data and from the higher resolution SWS spectra for the SWS field of view in both M 82 and NGC 253, indicating negligible contribution from the adjacent PAH $5.65 \mu \mathrm{m}$ feature and [Fe II] $5.34 \mu \mathrm{m}$ line in the lower resolution data. We measured the fluxes in the PAH features at 6.2, 7.7, 8.6, and $11.3 \mu \mathrm{m}$, in the [Ar II] $6.99 \mu \mathrm{m}$ line, and in the $\mathrm{PAH} 12.7 \mu \mathrm{m}+[\mathrm{Ne}$ II $] 12.81 \mu \mathrm{m}$ and PAH $15.7 \mu \mathrm{m}+\left[\mathrm{Ne}_{\mathrm{III}}\right]$ $15.56 \mu \mathrm{m}$ blends by integrating the flux under the feature profiles after subtracting a continuum baseline obtained by linear interpolation between adjacent spectral intervals. More sophisticated methods such as profile fitting (e.g. Uchida et al. 2000) are not necessary for our purposes and are difficult to apply to the lower signal-to-noise $(\mathrm{S} / \mathrm{N})$ ratio data of individual pixels in generating linemaps (Sect. 3.2).

We did not compute fluxes for the individual [ $\mathrm{Ne}$ II] $12.81 \mu \mathrm{m}$ and [Ne III] $15.56 \mu \mathrm{m}$ lines. The wavelength sampling is too coarse for reliable profile decomposition, with the feature peaks of each blend sampled by adjacent wavelength channels. In addition, our attempts to subtract the PAH contribution by attributing an excess in the blend $12.7 \mu \mathrm{m} / \mathrm{PAH} 11.3 \mu \mathrm{m}$ ratio to the $[\mathrm{Ne}$ II] $12.81 \mu \mathrm{m}$ line proved too sensitive to the definition of "pure" PAH $12.7 \mu \mathrm{m} / 11.3 \mu \mathrm{m}$ ratio (e.g. as measured outside of the starburst cores where comparatively little fine-structure line emission from $\mathrm{H}$ II regions is expected). Complications further arise from possible intrinsic variations in the PAH ratios, extinction effects $\left(A_{12.7 \mu \mathrm{m}} / A_{11.3 \mu \mathrm{m}} \approx 0.55\right)$, and unconstrained fine-structure line emission from disk $\mathrm{H}_{\text {II }}$ regions. The fluxes for [Ar II] $6.99 \mu \mathrm{m}$ are much more reliable because possible 
contributions by other features in our sample galaxies are substantially smaller, as mentioned above.

\subsection{Mid-infrared images: Mapping the features}

We obtained broad- and narrow-band images as well as maps of the PAHs and [Ar II] $6.99 \mu \mathrm{m}$ line emission from the ISOCAM data cubes by applying to each pixel the procedures described above for the spectra. Figures 5-7 present selected images and ratio maps for M 82, NGC 253, and NGC 1808. Contours corresponding to the same levels relative to the peak intensity are plotted for all continuum and emission feature maps for ease of comparison by visual inspection. A $3 \sigma$ contour is also shown to delineate regions where observed small-scale structures are reliable. In the following, we describe the various maps source by source; their interpretation will be discussed in subsequent sections.

\subsection{1. $\mathrm{M} 82$}

The continuum and emission feature maps in M82 show a globally smooth spatial distribution, centered and peaking roughly $5^{\prime \prime}$ southwest of the nucleus. The PAH and $5.5 \mu \mathrm{m}$ continuum emission are the most extended and symmetric, with disk-like isophotes elongated along the galactic plane $\left(\mathrm{PA} \approx 70^{\circ}\right.$. In contrast, the $15 \mu \mathrm{m}$ continuum and [Ar II] $6.99 \mu \mathrm{m}$ line emission have more compact distributions which are more asymmetric relative to the nucleus. The $15 \mu \mathrm{m} / 5.5 \mu \mathrm{m}$ ratio map outlines well the difference in peak morphology between the short and long wavelength continuum. The [Ar II] $6.99 \mu \mathrm{m}$ distribution is the most compact, with centroid (determined from the emission out to a radius of 25") displaced 3.5" southwest of that of the PAH and continuum emission, and showing only a slight extension towards the east.

Our ISOCAM maps provide an important complementary dataset to existing MIR images in the literature, which were mostly obtained in different bands or over limited regions (although with higher angular resolution up to $\approx 1^{\prime \prime}$ ). The distributions observed in our PAH maps and for the PAH $3.29 \mu \mathrm{m}$ feature by Normand et al. (1995) and Satyapal et al. (1995) at $\approx 1^{\prime \prime}$ resolution are consistent with each other. Our $15 \mu \mathrm{m}$ continuum map and the $19.2 \mu \mathrm{m}$ image of Telesco et al. (1991) are similar. Maps of the $N$-band $(10.8 \mu \mathrm{m})$ and $11.8-13.0 \mu \mathrm{m}$ emission generated from the ISOCAM data cubes agree well with those of Telesco et al. (1991) and Telesco \& Gezari (1992) within the regions covered by the latter two images.

Our [Ar II] $6.99 \mu \mathrm{m}$ linemap globally resembles those of other tracers of ionized gas from $\mathrm{H}$ II regions at mid- and nearinfrared wavelengths such as [Ne II] $12.81 \mu \mathrm{m}$, [Ar III] $8.99 \mu \mathrm{m}$, [S IV] $10.51 \mu \mathrm{m}, \mathrm{Br} \alpha, \mathrm{Br} \gamma$, and $\mathrm{Pa} \beta$ (Achtermann \& Lacy 1995; Satyapal et al. 1995). At a resolution of $1^{\prime \prime}-2^{\prime \prime}$, these maps reveal a rich sub-structure dominated by prominent sources $\approx 6^{\prime \prime}$ and $12^{\prime \prime}$ southwest of the nucleus and $\approx 6^{\prime \prime}$ to the northeast (labeled W1, W2, and E1 by Achtermann \& Lacy 1995). While their intensity ratio depends somewhat on the emission line considered, $\mathrm{W} 1$ and $\mathrm{W} 2$ are together about three times brighter than E1. Radial velocity data of the [Ne II] $12.81 \mu \mathrm{m}$ and $\mathrm{Br} \gamma$ emission (Larkin et al. 1994; Achtermann \& Lacy 1995) suggest that most sources reside in a nearly edge-on rotating ring at radius coinciding in projection with $\mathrm{W} 1$ and $\mathrm{E} 1$ as well as along the stellar bar at larger radii, where the most recent starburst episode took place about $5 \mathrm{Myr}$ ago (e.g. Förster Schreiber et al. 2003). Within the positional uncertainties and resolution limitations, the spatial distribution of our [Ar II] $6.99 \mu \mathrm{m}$ map peaks between $\mathrm{W} 1$ and $\mathrm{W} 2$ and encompasses $\mathrm{E} 1$, and thus traces well the youngest starburst regions.

Little differences are seen between the PAH maps but the PAH $6.2 \mu \mathrm{m} / 7.7 \mu \mathrm{m}$ and PAH $8.6 \mu \mathrm{m} / 11.3 \mu \mathrm{m}$ ratio maps reveal spatial variations at the $20 \%$ and $60 \%$ level, respectively. The variations are significant within the brighter emission regions along the disk, where the relative uncertainties are $<15 \%$. Structures seen towards the map edges are much less reliable as they become comparable in amplitude to the uncertainties. The PAH $6.2 \mu \mathrm{m} / 7.7 \mu \mathrm{m}$ ratio is lower along the disk and reaches minima on each side of the nucleus. The overall morphology appears to curve northwards away from the nucleus and extensions are hinted at above and below the galactic plane. Similar shape and spurs are observed notably in the large-scale distribution of the molecular and ionized gas line emission and of the radio continuum emission (e.g. Shen \& Lo 1995; Achtermann \& Lacy 1995; Wills et al. 1999). The spatial variations in the PAH $8.6 \mu \mathrm{m} / 11.3 \mu \mathrm{m}$ ratio match roughly those of the PAH $6.2 \mu \mathrm{m} / 7.7 \mu \mathrm{m}$ ratio, with higher values along the disk and maxima flanking the nucleus. Noticeably, the western peak lies closer to the nucleus than the western PAH $6.2 \mu \mathrm{m} / 7.7 \mu \mathrm{m}$ minimum, and the apparent curving and large-scale extensions have no counterpart in the PAH $8.6 \mu \mathrm{m} / 11.3 \mu \mathrm{m}$ map.

Figure 8 compares the PAH ratio maps with the $\mathrm{CO} \mathrm{J:1 \rightarrow}$ 0 millimetric emission of Shen \& Lo (1995) convolved at the resolution of the ISOCAM maps. The overall correspondence of the PAH $6.2 \mu \mathrm{m} / 7.7 \mu \mathrm{m}$ minima and PAH $8.6 \mu \mathrm{m} / 11.3 \mu \mathrm{m}$ maxima with the peaks in $\mathrm{CO}$ emission, as well as the curved shape and northeastern extension for the PAH $6.2 \mu \mathrm{m} / 7.7 \mu \mathrm{m}$ ratio, is quite striking. We believe that the observed variations in PAH ratios are mostly real. Characteristic patterns expected for ghosts are not seen (unresolved ring- or arclike features most prominent in the presence of point-like sources). Artifacts due to the uncorrected flat field and straylight could produce extended lobes on each side of an axis at $\mathrm{PA} \approx 150^{\circ}$ for the M 82 data, i.e. roughly the minor axis (Biviano et al. 1998a, 1998b; Okumura 2000). However, such lobes would have a much larger extent than the features seen in our PAH ratio maps and the chromatic dependence between 6.2 and $7.7 \mu \mathrm{m}$, and 8.6 and $11.3 \mu \mathrm{m}$ is predicted and observed to be smaller than the measured variations at $20 \%$ and $60 \%$ levels, respectively.

\subsubsection{NGC 253}

In NGC 253, the emission in the continuum bands, PAH features, and [Ar II] $6.99 \mu \mathrm{m}$ line is characterized by a strong peak within $1^{\prime \prime}-2^{\prime \prime}$ of the nucleus, embedded in a diffuse envelope elongated along the galactic plane $\left(\mathrm{PA} \approx 50^{\circ}\right)$. The $15 \mu \mathrm{m}$ continuum and [Ar II] $6.99 \mu \mathrm{m}$ line distributions do not seem to 
Table 3. Intervals used to measure the continuum and features.

\begin{tabular}{|c|c|c|c|}
\hline Property & Symbol & $\begin{array}{l}\text { Continuum points or intervals } \\
\qquad(\mu \mathrm{m})\end{array}$ & $\begin{array}{l}\text { Integration limits } \\
\qquad(\mu \mathrm{m})\end{array}$ \\
\hline PAH emission $^{a}$ & $f_{\mathrm{PAH}}$ & $\ldots$ & $6.00-9.00$ \\
\hline VSG emission $^{b}$ & $f_{\mathrm{VSG}}$ & $\ldots$ & $13.5-15.0$ \\
\hline $5.5 \mu \mathrm{m}$ continuum & $f_{5.5 \mu \mathrm{m}}$ & $\ldots$ & $5.40-5.52$ \\
\hline $15.0 \mu \mathrm{m}$ continuum & $f_{15 \mu \mathrm{m}}$ & $\ldots$ & $14.8-15.2$ \\
\hline PAH $6.2 \mu \mathrm{m}$ & $F_{6.2}$ & $5.81-5.99,6.57-6.80$ & $6.04-6.51$ \\
\hline $\mathrm{PAH} 7.7 \mu \mathrm{m}$ & $F_{7.7}$ & $7.14,8.22-8.38$ & $7.19-8.17$ \\
\hline РAH $8.6 \mu \mathrm{m}$ & $F_{8.6}$ & $8.27-8.33,8.84-8.89$ & $8.33-8.84$ \\
\hline PAH $11.3 \mu \mathrm{m}$ & $F_{11.3}$ & $10.95,11.7-11.8$ & $11.1-11.7$ \\
\hline $\mathrm{PAH} 12.7 \mu \mathrm{m}+[\mathrm{Ne} \mathrm{II}] 12.81 \mu \mathrm{m}$ & $F_{12.7}$ & $12.1-12.2,13.2-13.3$ & $12.3-13.2$ \\
\hline PAH $15.7 \mu \mathrm{m}+[\mathrm{Ne}$ III] $15.56 \mu \mathrm{m}$ & $F_{15.6}$ & $15.0-15.2,16.0-16.1$ & $15.3-15.9$ \\
\hline [Ar II] $6.99 \mu \mathrm{m}$ & $F_{[\mathrm{ArII}]}$ & $6.74-6.86,7.14-7.19$ & $6.86-7.14$ \\
\hline
\end{tabular}

${ }^{a}$ Integrated flux in the band including the short wavelength PAH emission complex.

${ }^{b}$ Integrated flux in the band probing the continuum emission from VSGs free from strong emission lines and PAH features.

Table 4. Broad- and narrow-band measurements ${ }^{a}$.

\begin{tabular}{llllll}
\hline \hline Source & Region & $f_{\mathrm{PAH}}$ & $f_{\mathrm{VSG}}$ & $f_{5.5 \mu \mathrm{m}}$ & $f_{15 \mu \mathrm{m}}$ \\
\hline M 82 & CAM FOV & $61.1 \pm 0.8$ & $78.3 \pm 0.3$ & $6.19 \pm 1.98$ & $80.8 \pm 0.6$ \\
& Disk & $12.7 \pm 0.1$ & $11.3 \pm 0.1$ & $1.16 \pm 0.17$ & $10.8 \pm 0.1$ \\
& Core & $26.9 \pm 0.1$ & $45.7 \pm 0.3$ & $3.15 \pm 0.08$ & $49.3 \pm 0.5$ \\
& MIR Peak & $8.03 \pm 0.06$ & $16.3 \pm 0.2$ & $1.02 \pm 0.04$ & $17.9 \pm 0.4$ \\
NGC 253 & CAM FOV & $11.9 \pm 0.3$ & $25.3 \pm 0.2$ & $2.12 \pm 1.31$ & $27.2 \pm 0.3$ \\
& Disk & $2.49 \pm 0.03$ & $3.93 \pm 0.03$ & $0.41 \pm 0.19$ & $4.12 \pm 0.06$ \\
& Core & $5.63 \pm 0.03$ & $15.3 \pm 0.1$ & $1.05 \pm 0.11$ & $16.8 \pm 0.3$ \\
& MIR Peak & $1.68 \pm 0.02$ & $5.74 \pm 0.09$ & $0.35 \pm 0.02$ & $6.40 \pm 0.19$ \\
NGC 1808 & Core & $2.66 \pm 0.05$ & $3.13 \pm 0.04$ & $\ldots$ & $3.08 \pm 0.07$ \\
& MIR Peak & $1.10 \pm 0.01$ & $1.39 \pm 0.03$ & $0.11 \pm 0.01$ & $1.41 \pm 0.05$ \\
\hline
\end{tabular}

a All flux densities are expressed in Jy. The uncertainties result from the formal effective uncertainties of the relative fluxes (see Sect. 2).

Table 5. Emission feature measurements ${ }^{a}$.

\begin{tabular}{|c|c|c|c|c|c|c|c|c|}
\hline Source & Region & $F_{6.2}$ & $F_{7.7}$ & $F_{8.6}$ & $F_{11.3}$ & $F_{12.7}$ & $F_{15.6}$ & $F_{[\mathrm{ArII}]}$ \\
\hline \multirow[t]{4}{*}{ M 82} & CAM FOV & $103 \pm 1$ & $226 \pm 1$ & $31.8 \pm 2.4$ & $57.7 \pm 0.9$ & $54.0 \pm 0.4$ & $5.77 \pm 0.47$ & $6.05 \pm 0.31$ \\
\hline & Disk & $21.5 \pm 0.2$ & $46.5 \pm 0.2$ & $6.70 \pm 0.08$ & $13.1 \pm 0.1$ & $9.74 \pm 0.06$ & $0.76 \pm 0.02$ & $0.67 \pm 0.02$ \\
\hline & Core & $43.7 \pm 0.5$ & $100 \pm 1$ & $13.8 \pm 0.4$ & $22.0 \pm 0.5$ & $26.4 \pm 0.5$ & $3.49 \pm 0.39$ & $3.96 \pm 0.15$ \\
\hline & MIR Peak & $12.8 \pm 0.3$ & $29.7 \pm 0.4$ & $3.94 \pm 0.15$ & $5.76 \pm 0.25$ & $8.50 \pm 0.35$ & $1.43 \pm 0.21$ & $1.64 \pm 0.08$ \\
\hline \multirow[t]{4}{*}{ NGC 253} & CAM FOV & $16.1 \pm 1.1$ & $36.3 \pm 0.3$ & $4.24 \pm 0.15$ & $10.2 \pm 0.2$ & $10.6 \pm 0.2$ & $0.55 \pm 0.12$ & $1.93 \pm 0.12$ \\
\hline & Disk & $3.55 \pm 0.09$ & $7.63 \pm 0.07$ & $1.03 \pm 0.02$ & $2.41 \pm 0.03$ & $2.02 \pm 0.02$ & $0.077 \pm 0.007$ & $0.36 \pm 0.01$ \\
\hline & Core & $7.30 \pm 0.11$ & $17.6 \pm 0.2$ & $1.86 \pm 0.05$ & $4.09 \pm 0.11$ & $5.71 \pm 0.17$ & $0.37 \pm 0.10$ & $1.13 \pm 0.03$ \\
\hline & MIR Peak & $2.02 \pm 0.06$ & $4.95 \pm 0.09$ & $0.54 \pm 0.02$ & $1.07 \pm 0.06$ & $1.83 \pm 0.10$ & $0.19 \pm 0.05$ & $0.37 \pm 0.02$ \\
\hline \multirow[t]{2}{*}{ NGC 1808} & Core & $4.17 \pm 0.19$ & $9.39 \pm 0.12$ & $1.38 \pm 0.03$ & $3.32 \pm 0.08$ & $3.05 \pm 0.06$ & $0.096 \pm 0.016$ & $0.23 \pm 0.02$ \\
\hline & MIR Peak & $1.59 \pm 0.05$ & $3.74 \pm 0.08$ & $0.57 \pm 0.02$ & $1.31 \pm 0.05$ & $1.34 \pm 0.04$ & $0.039 \pm 0.008$ & $0.087 \pm 0.007$ \\
\hline
\end{tabular}

a All fluxes are expressed in $10^{-14} \mathrm{~W} \mathrm{~m}^{-2}$. The uncertainties result from the formal effective uncertainties of the relative fluxes (see Sect. 2).

extend as far as the PAH emission in the outer parts of the source. The noisy $\lambda<6 \mu \mathrm{m}$ channels prevent reliable assessment of the lower level, large-scale $5.5 \mu \mathrm{m}$ continuum emission. The centroids in the various images are essentially indistinguishable (differences $<0.5$ pixel).

Though the images reveal little spatial structure because of the intrinsically small source size and the limited angular resolution, they are consistent with expectations at this resolution based on previously published MIR maps (mostly obtained at $\sim 1^{\prime \prime}$ resolution). These include broad-band images at 10.8 ( $N$ band) and $19.2 \mu \mathrm{m}$, narrow-band images at 8.5, 10.0, 12.5 , and $20.2 \mu \mathrm{m}$ tracing $\mathrm{PAH}$ and/or continuum emission, maps of the 3.29 and $11.3 \mu \mathrm{m}$ PAH emission, and images of the [Ne II] $12.81 \mu \mathrm{m}$ line and of the underlying continuum $+\mathrm{PAH}$ 
emission (Piña et al. 1992; Telesco et al. 1993; Keto et al. 1993, 1999; Kalas \& Wynn-Williams 1994; Böker et al. 1998). We note that the [Ne II] linemaps of Böker et al. (1998) and Keto et al. (1999) likely contain a contribution from PAH $12.7 \mu \mathrm{m}$ emission because the observations were made at low spectral resolution $(F W H M \approx 0.2 \mu \mathrm{m})$.

The spatial distribution seen in all our maps is evidently dominated by a very prominent compact MIR source, better outlined in arcsecond resolution images. Keto et al. (1999) associated this source with a bright off-nucleus super star cluster resolved by optical Hubble Space Telescope observations. Based on their data, the source has a size of $20 \mathrm{pc}$ (marginally resolved) and accounts for $\approx 20 \%$ of the total continuum emission at 12 and $20 \mu \mathrm{m}$, and $12 \%$ of the total [Ne II] $12.81 \mu \mathrm{m}$ flux. From our own measurements, nearly $25 \%$ of the total continuum emission detected with ISOCAM between 13.5 and $15 \mu \mathrm{m}$ originates in the $70 \mathrm{pc}$-diameter MIR peak; this fraction varies between $10 \%$ and $30 \%$ for our various broad- and narrow-band and emission feature measurements (Tables 4 and 5). NGC 253 is quite remarkable in the compactness of its main MIR emitting region, with a $F W H M \lesssim 150 \mathrm{pc}$ while the optical disk extends over $\sim 20 \mathrm{kpc}$. Although of a different nature, the interacting system NGC 4038/4039 offers a similar example, with $15 \%$ of its $12.5-18 \mu \mathrm{m}$ luminosity being produced within a $100 \mathrm{pc}-$ size star-forming knot in the overlapping region between the galaxies (Mirabel et al. 1998).

Additional small-scale structure of NGC 253 at MIR wavelengths includes a secondary much fainter peak in the 12 and $20 \mu \mathrm{m}$ continuum emission nearly coinciding with the nucleus, about $2^{\prime \prime}$ northeast of the prominent source discussed above. The [Ne II] $12.81 \mu \mathrm{m}$ line emission differs somewhat from the continuum, except possibly for the brightest peak, showing a bilobal or arc-like structure also hinted at in $\mathrm{Br} \gamma$ images and suggestive of a circumnuclear star-forming ring (Böker et al. 1998; Engelbracht et al. 1998; Keto et al. 1999). None of these features, however, is resolved with ISOCAM.

The PAH $6.2 \mu \mathrm{m} / 7.7 \mu \mathrm{m}$ and PAH $8.6 \mu \mathrm{m} / 11.3 \mu \mathrm{m}$ ratio maps show variations of about $40 \%$ and nearly a factor of 2 , respectively. These are statistically significant in view of the corresponding formal uncertainties of $\leq 20 \%$ and $<30 \%$. The ratio maps differ markedly, with the PAH $6.2 \mu \mathrm{m} / 7.7 \mu \mathrm{m}$ image indicating a general increase from south to north of the nucleus whereas the PAH $8.6 \mu \mathrm{m} / 11.3 \mu \mathrm{m}$ ratio appears more centrally concentrated, peaking near the nucleus and slightly more extended northeast. The reality of the arc-like feature $\approx 10^{\prime \prime}$ northwest of the nucleus in the PAH $8.6 \mu \mathrm{m} / 11.3 \mu \mathrm{m}$ map is dubious because it is barely resolved and could be due to ghost effects given the strong unresolved peak of the emission. No corresponding structure that could perhaps support this feature is seen in maps of the molecular gas emission, of the radio continuum emission, of tracers of ionized gas or even of extinction (e.g. Peng et al. 1996; Ulvestad \& Antonucci 1997; Engelbracht et al. 1998). The limited region for which $S / N \geq 3$ prevents us from examining the larger-scale distribution in PAH ratios.

\subsubsection{NGC 1808}

The starburst in NGC 1808 covers a region of comparable physical size to that of $\mathrm{M} 82$ but being three times more distant, less structural details are resolved by ISOCAM. The PAH emission appears the most extended and oriented parallel to the major axis of the galaxy $\left(\mathrm{PA} \approx 140^{\circ}\right)$, peaking at the nucleus. The $15 \mu \mathrm{m}$ emission follows closely the PAH emission. The [Ar II] $6.99 \mu \mathrm{m}$ line emission is the most distinct in that the peak clearly is off-nucleus, about 5 " to the southeast, and the centroid of the emission region lies $2.5^{\prime \prime}$ southeast of that for the $15 \mu \mathrm{m}$ continuum and PAH emission. The [Ar II] $6.99 \mu \mathrm{m}$ map agrees well in peak position and extent with the global distribution of the most intense star-forming regions, or hot spots, as traced by $\mathrm{H}$ recombination lines and radio continuum emission (e.g. Saikia et al. 1990; Krabbe et al. 1994; Kotilainen et al. 1996). Due to the limited number of pixels with $S / N \geq 3$, no useful ratio maps could be made for NGC 1808 .

Previously published $N$ band images of NGC 1808 (Telesco et al. 1993; Krabbe et al. 2001) show an overall similar morphology as our $15 \mu \mathrm{m}$ continuum and PAH feature maps with, at $\approx 1^{\prime \prime}$ resolution, a strong point-like source at the nucleus and a southeastern extension covering the starburst regions. ISOCAM broad-band LW4 $(6 \mu \mathrm{m})$ polarisation observations were presented by Siebenmorgen et al. (2001) along with a CVF spectrum of the central $25^{\prime \prime}$ of NGC 1808 which is essentially identical in shape to ours of the starburst core (30" aperture; Fig. 3). Siebenmorgen et al. (2001) assigned all detected features to PAHs and successfully reproduced them with $\mathrm{PAH}$ emission alone in their radiative transfer models. As also noted by these authors, the contribution of nebular gas emission lines blended with PAH features is uncertain for NGC 1808 , and we cannot constrain it using, e.g., SWS data as for M 82 and NGC 253. However, our [Ar II] $6.99 \mu \mathrm{m}$ map clearly differs from those of the PAH emission and is consistent with the spatial distribution of the bulk of $\mathrm{H}_{\text {II }}$ regions, supporting the idea that the $7 \mu \mathrm{m}$ feature is indeed dominated by the [Ar II] line instead of PAHs.

\subsection{Salient features}

We summarize here the most important aspects of the results presented above:

- in terms of features present, the MIR spectral energy distribution is essentially identical among the three galaxies as well as within each of them,

- the largest spectral variations are observed in the $\lambda \gtrsim$ $11 \mu \mathrm{m}$ continuum intensity relative to the shorter wavelength emission, while the shape of the $\lambda=5-11 \mu \mathrm{m}$ region is nearly invariant,

- the PAH emission is spatially more extended than the $15 \mu \mathrm{m}$ continuum and [Ar II] $6.99 \mu \mathrm{m}$ line emission,

- the [Ar II] $6.99 \mu \mathrm{m}$ line emission traces well the young starburst regions, and

- the relative intensities of the PAH features can exhibit complex spatial variations. 


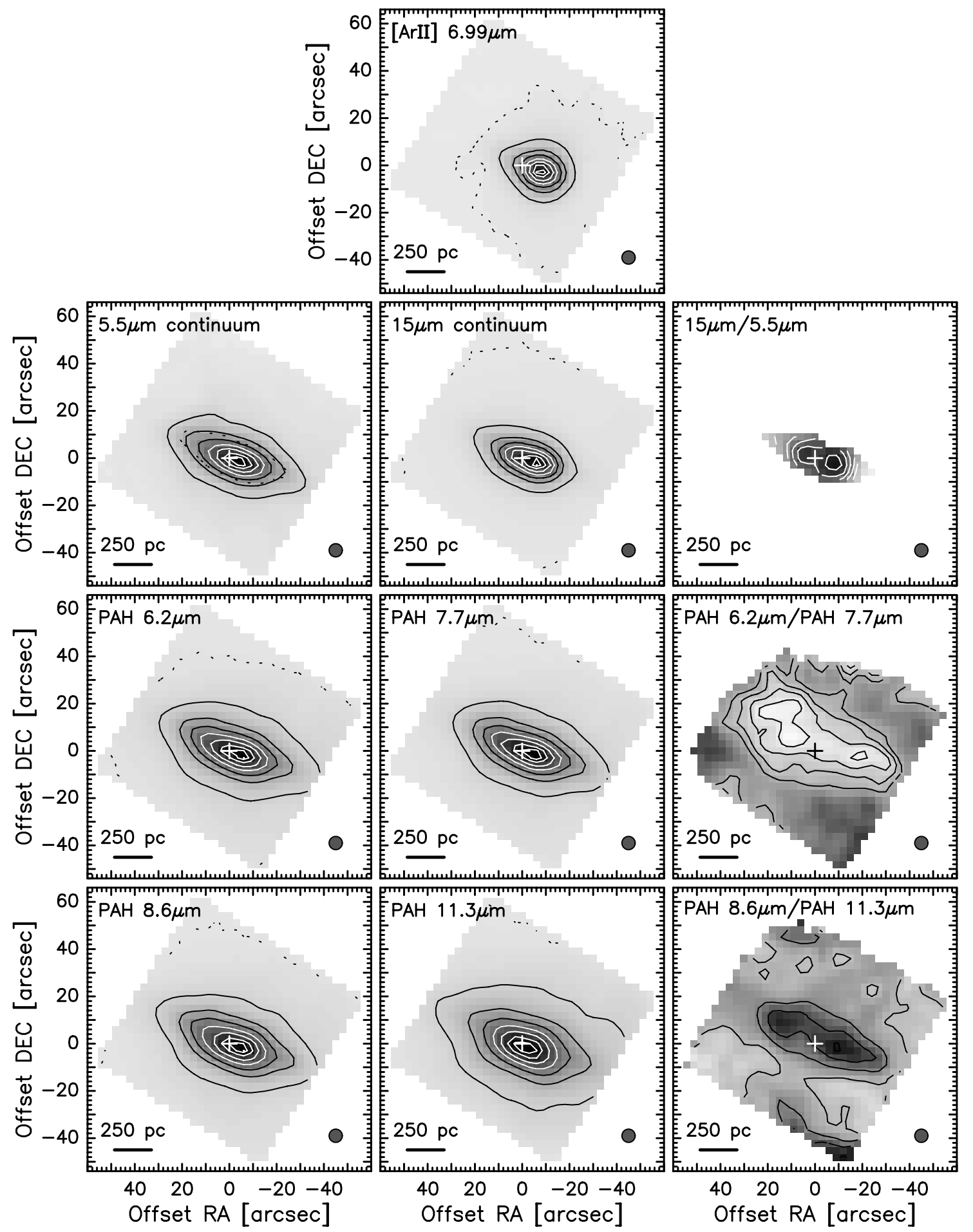

Fig. 5. Selected ISOCAM maps of M 82. The maps are identified at the top of each panel while the physical linear scale and the $F W H M$ of the PSF are indicated at the bottom left and right. The grayscale is linear, with white and black tones corresponding to the minimum and maximum values displayed. For the continuum and emission feature images, the solid contours are $10 \%, 25 \%, 40 \%, 55 \%, 70 \%, 85 \%$, and $95 \%$ of the maximum fluxes. For the 5.5 and $15.0 \mu \mathrm{m}$ continuum maps, the peaks are 10.3 and $187 \mathrm{mJy} \operatorname{arcsec}^{-2}$, respectively. For the [ArII] $6.99 \mu \mathrm{m}$ and the PAH 6.2, 7.7, 8.6, and $11.3 \mu \mathrm{m}$ maps, they are 2.05, 12.5, 28.9, 3.84, and 5.54, respectively, in units of $10^{-16} \mathrm{~W} \mathrm{~m}^{-2}$ arcsec $^{-2}$. The contour levels for the ratio maps are as follows: from 10 to 18 in steps of 2 for the $15.0 \mu \mathrm{m} / 5.5 \mu \mathrm{m}$ continuum ratio, from 0.42 to 0.48 in steps of 0.02 for the PAH $6.2 \mu \mathrm{m} / 7.7 \mu \mathrm{m}$ ratio, and from 0.45 to 0.75 in steps of 0.10 for the PAH $8.6 \mu \mathrm{m} / 11.3 \mu \mathrm{m}$ ratio. The dotted contours in the continuum and feature maps enclose the regions where data values exceed $3 \sigma$, and ratio maps are displayed for the area where this is satisfied by both images involved. The cross at relative coordinates $\left(0^{\prime \prime}, 0^{\prime \prime}\right)$ indicates the location of the galaxy nucleus. 

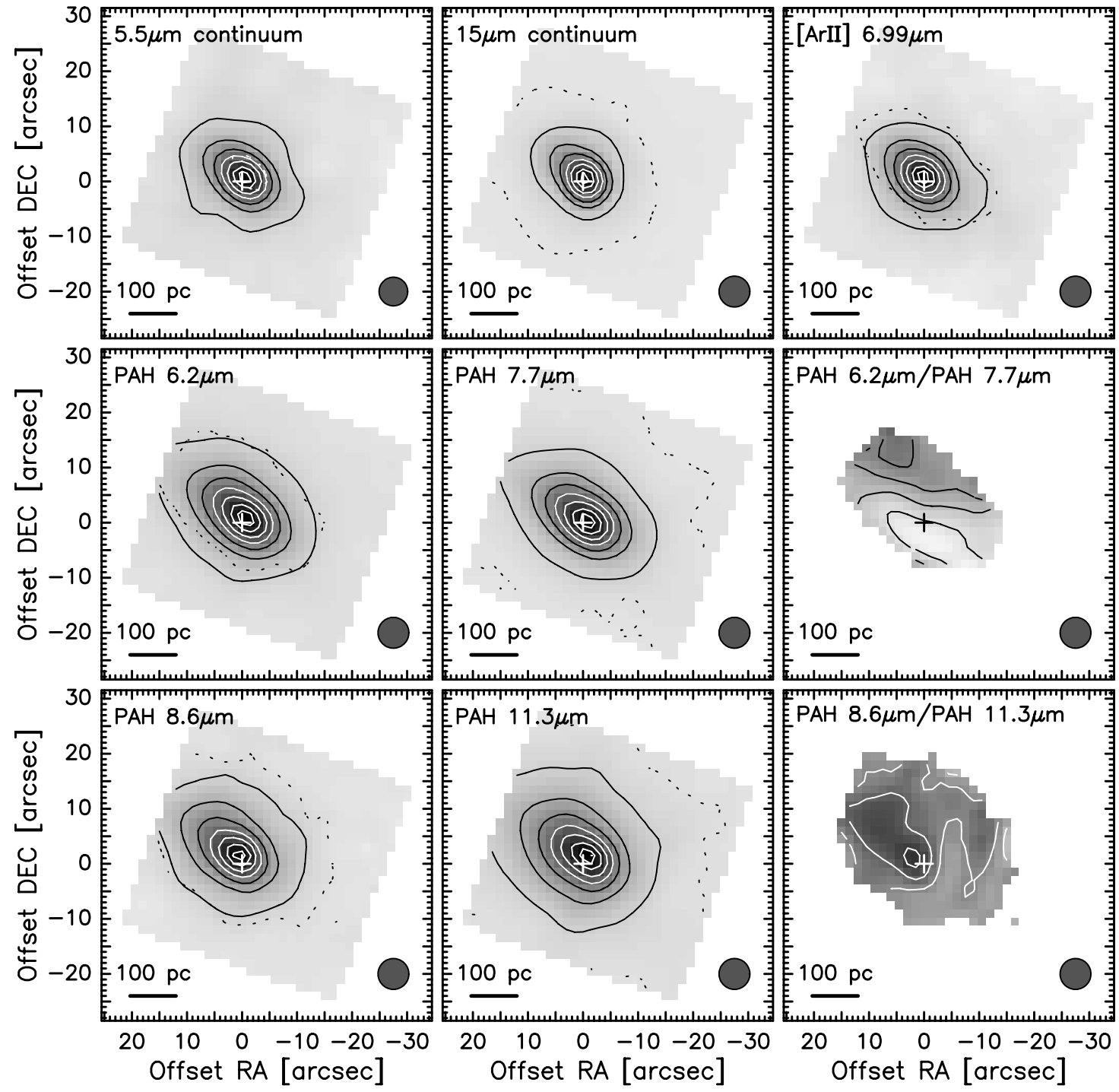

Fig. 6. Selected ISOCAM maps of NGC 253. The maps are identified at the top of each panel while the physical linear scale and the $F W H M$ of the PSF are indicated at the bottom left and right. The grayscale is linear, with white and black tones corresponding to the minimum and maximum values displayed. For the continuum and emission feature images, the contours are 10\%, 25\%, 40\%, 55\%, 70\%, 85\%, and 95\% of the maximum fluxes. For the 5.5 and $15.0 \mu \mathrm{m}$ continuum maps, the peaks are 13.6 and $269 \mathrm{mJy} \operatorname{arcsec}^{-2}$. For the [ArII] $6.99 \mu \mathrm{m}$ and the PAH 6.2, 7.7, 8.6, and $11.3 \mu \mathrm{m}$ maps, they are $1.50,7.66,19.1,2.33$, and 4.08, respectively, in units of $10^{-16} \mathrm{~W} \mathrm{~m}^{-2} \operatorname{arcsec}^{-2}$. The contour levels for the ratio maps are as follows: from 0.40 to 0.55 in steps of 0.05 for the PAH $6.2 \mu \mathrm{m} / 7.7 \mu \mathrm{m}$ ratio, and from 0.30 to 0.60 in steps of 0.10 for the PAH $8.6 \mu \mathrm{m} / 11.3 \mu \mathrm{m}$ ratio. The dotted contours in the continuum and feature maps enclose the regions where data values exceed $3 \sigma$ (for the $5.5 \mu \mathrm{m}$ continuum map, the $3 \sigma$ limit lies between the contours of $55 \%$ and $70 \%$ of maximum flux), and ratio maps are displayed for the area where this is satisfied by both images involved. The cross at relative coordinates $\left(0^{\prime \prime}, 0^{\prime \prime}\right)$ indicates the location of the galaxy nucleus.

\section{Origin and spatial distribution of the PAH and continuum emission}

The ubiquity of PAH features - seen towards sources as diverse as circumstellar regions, diffuse atomic clouds, $\mathrm{H}_{\text {II }}$ regions, molecular clouds, normal galaxies, starbursts, and ULIRGs and the close association of the MIR VSG continuum emission with Galactic and extragalactic actively star-forming sites now seem well established; the ISO mission played a major role in this recognition (see Geballe 1997; Tokunaga 1997; Tielens et al. 1999; Cesarsky \& Sauvage 1999; Genzel \& Cesarsky 2000 for reviews). Spatial mapping of Galactic sources has revealed in more detail the origin of the main MIR emission components in star-forming regions: PAH features arise predominantly in the photodissociation regions (PDRs) at the interface between $\mathrm{H}_{\text {II }}$ regions and molecular clouds while the VSG $\lambda \gtrsim 11 \mu \mathrm{m}$ continuum is more intense and steeper closer to the exciting source (e.g. Verstraete et al. 1996; Cesarsky et al. 1996a, 1996b; Crété et al. 1999). By extension, in starforming galaxies, the emission components are believed to trace PDRs and $\mathrm{H}_{\text {II }}$ regions, respectively. This is supported by spectral decomposition of starburst galaxies (Tran 1998; Laurent et al. 2000), and by photometric and spectrophotometric imaging of spiral galaxies and starburst systems (e.g. Mirabel et al. 1998; Roussel et al. 2001a). Low excitation finestructure line emission such as [Ar II] $6.99 \mu \mathrm{m}$, [Ar III] $8.99 \mu \mathrm{m}$, 


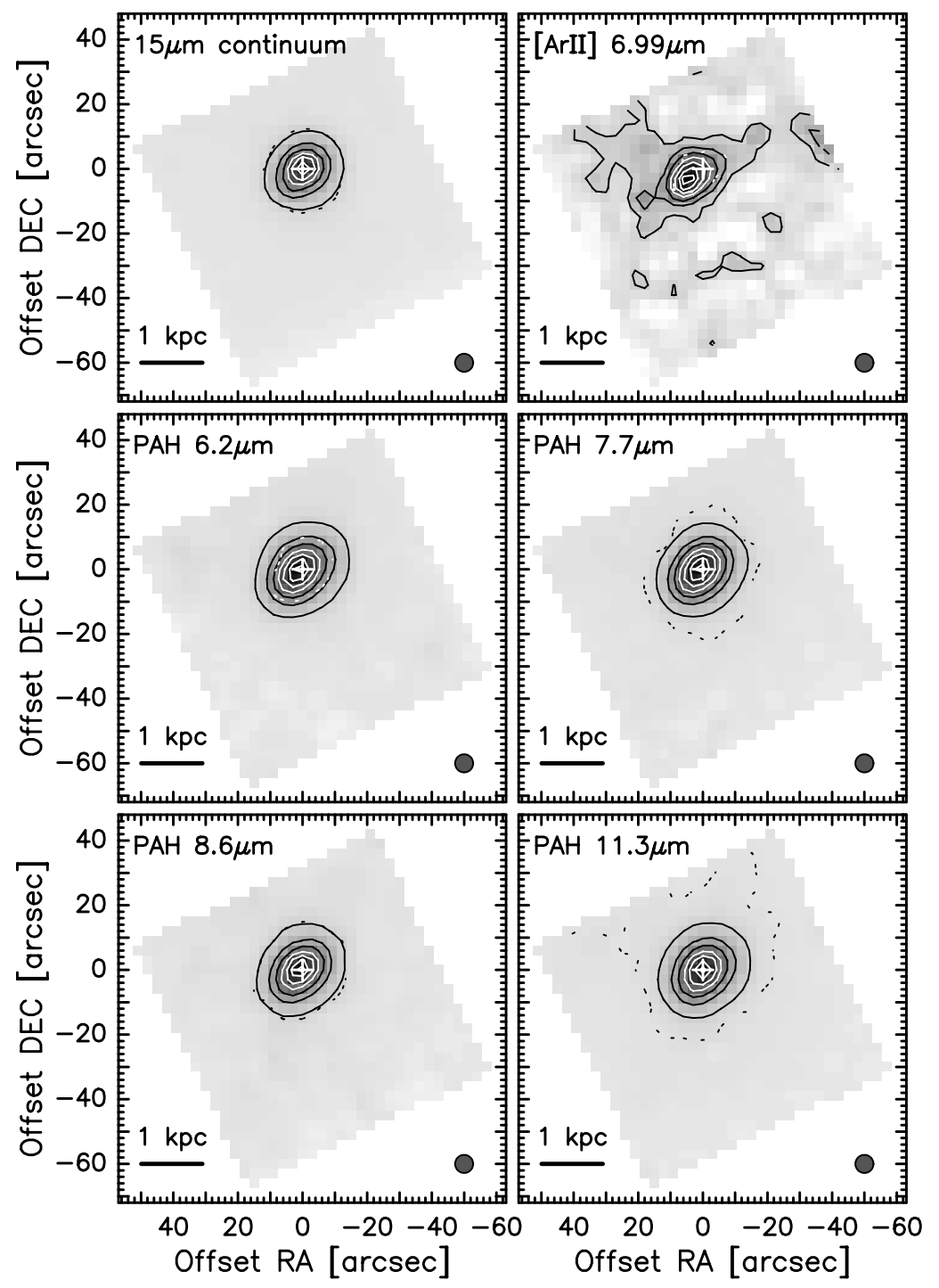

Fig. 7. Selected ISOCAM maps of NGC 1808. The maps are identified at the top of each panel while the physical linear scale and the $F W H M$ of the PSF are indicated at the bottom left and right. The grayscale is linear, with white and black tones corresponding to the minimum and maximum values displayed. The contours correspond to $10 \%, 25 \%, 40 \%, 55 \%, 70 \%, 85 \%$, and $95 \%$ of the maximum fluxes. For the $15.0 \mu \mathrm{m}$ continuum map, the peak is $18.7 \mathrm{mJy} \mathrm{arcsec}^{-2}$. For the [ArII] $6.99 \mu \mathrm{m}$ and the PAH 6.2, 7.7, 8.6, and $11.3 \mu \mathrm{m}$ maps, they are $0.13,1.82,4.23$, 0.71 , and 1.58 , respectively, in units of $10^{-16} \mathrm{~W} \mathrm{~m}^{-2} \operatorname{arcsec}^{-2}$. The dotted contours in the continuum and feature maps enclose the regions where data values exceed $3 \sigma$. The cross at relative coordinates $\left(0^{\prime \prime}, 0^{\prime \prime}\right)$ indicates the location of the galaxy nucleus.

[Ne II] $12.81 \mu \mathrm{m}$, and [Ne III] $15.56 \mu \mathrm{m}$ usually accompanies the long-wavelength VSG continuum and originates primarily in H il regions (e.g. Sturm et al. 2000).

The interpretation of the ISOCAM maps of M82, NGC 253, and NGC 1808 within the above framework is not straightforward. Figure 9 complements the various maps with light profiles of the $6-9 \mu \mathrm{m}, 13.5-15 \mu \mathrm{m}$, and [Ar II] $6.99 \mu \mathrm{m}$ emission taken along the major and minor axes of the galaxies. The profiles clearly support the extension along the major axis indicated by the maps, and show that the emission in these tracers along the minor axis is but marginally resolved and essentially indistinguishable within each galaxy.

Somewhat surprisingly given their common origin in $\mathrm{H}_{\mathrm{II}}$ regions, a close spatial correlation between the VSG continuum and the [Ar II] $6.99 \mu \mathrm{m}$ line emission is not observed in our sample galaxies. The exception may be NGC 253 but this probably results from the intrinsically small MIR source size and resolution limitations (Sect. 3.2.2). Although the extension to the northeast is real, the differences between the various profiles even along the major axis are smaller than the PSF FWHM. We conclude for NGC 253 that on scales comparable to and larger than the resolution of our ISOCAM maps $\left(5.2^{\prime \prime}\right.$, or $\left.\approx 60 \mathrm{pc}\right)$, no significant differences are observed in the spatial distribution of the PAH, VSG, and [Ar II] $6.99 \mu \mathrm{m}$ emission within the central $400 \mathrm{pc}$.

For M 82, the [ArII] $6.99 \mu \mathrm{m}$ emission along the galactic plane peaks at a different position and is more compact than the VSG and PAH emission. The different distributions of the VSG and [Ar II] $6.99 \mu \mathrm{m}$ emission could be interpreted in terms of differences in average energy of the exciting photons: the [Ar II] $6.99 \mu \mathrm{m}$ line traces the $\geq 16 \mathrm{eV}$ radiation field while the VSGs may be heated by UV photons at lower energies as well 


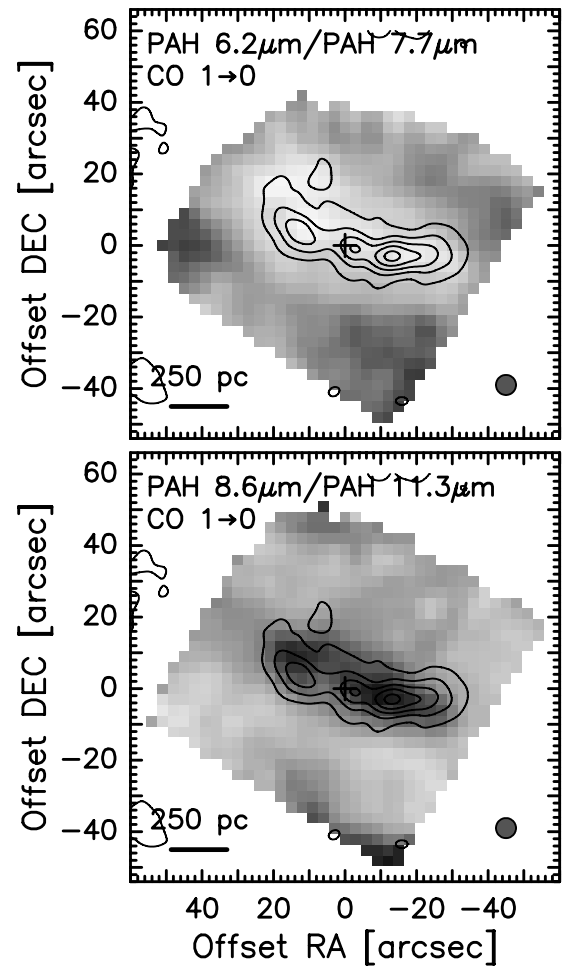

Fig. 8. Comparison of ISOCAM PAH ratio maps of M 82 with the distribution of $\mathrm{CO} J: 1 \rightarrow 0$ millimetric emission. The PAH $6.2 \mu \mathrm{m} / 7.7 \mu \mathrm{m}$ and PAH $8.6 \mu \mathrm{m} / 11.3 \mu \mathrm{m}$ maps (top and bottom panels, respectively) are displayed as grayscale images as for Fig. 5. The contours show the CO $J: 1 \rightarrow 0$ emission from the map of Shen \& Lo (1995) convolved at the resolution of the ISOCAM images (from the original $2.5^{\prime \prime}$ to $5.6^{\prime \prime}$ ). The physical linear scale and the FWHM of the ISOCAM PSF are indicated at the bottom left and right. The cross at relative coordinates $\left(0^{\prime \prime}, 0^{\prime \prime}\right)$ marks the location of the galaxy nucleus.

(Désert et al. 1990; Siebenmorgen \& Krügel 1992; Boulanger et al. 1994; Dwek et al. 1997; Jones \& d'Hendecourt 2000). The brighter PAH emission around the nucleus could be originating predominantly in the PDRs associated with the H II regions within the most active starburst sites. Beyond a radius of $\approx 300 \mathrm{pc}$, the PAH profile is resolved out from the VSG profile and more extended. These differences could be attributed to excitation of aromatic band carriers by yet softer photons or to an increased filling factor for the PDRs compared to the H II regions in less intense starburst regions, and perhaps to a contribution from diffuse cirrus clouds as observed in the Milky Way and in some spiral galaxies (e.g. Ristorcelli et al. 1994; Onaka et al. 1996; Mattila et al. 1999; Roussel et al. 2001a; Li \& Draine 2002). Alternative possibilities could include destruction by photodissociation or depletion of the ISM in PAHs and VSGs in the most intense starburst regions, flattening the spatial distribution around the peak (e.g. Boulanger et al. 1988; Carral et al. 1994; Normand et al. 1995).

In NGC 1808, the bulk of the [Ar II] $6.99 \mu \mathrm{m}$ emission is clearly displaced to the southeast compared to the emission in the PAH and VSG bands defined above, which have virtually identical distributions. As discussed in Sect. 3.2.3, the [Ar II] $6.99 \mu \mathrm{m}$ distribution agrees well with the off-nucleus location of the main star-forming sites. The spectrum integrated over the starburst core and MIR peak in NGC 1808 is also much flatter than observed towards the corresponding regions in M 82 and NGC 253 and resembles more the spectrum of their outer disks (Fig. 3). The long-wavelength continuum in NGC 1808 may in fact not be dominated by VSG emission from pure $\mathrm{H}_{\text {II }}$ regions but rather produced primarily in PDRs by small particles akin to the carriers of the main $U I B$ bands. This is reminiscent of the situation in disks of spiral galaxies. There, the $15 \mu \mathrm{m}$ emission correlates with the $7 \mu \mathrm{m}$ PAH-dominated emission and lies in a distinct regime compared to more active regions including circumnuclear regions in spiral (especially barred) galaxies and starbursts, characterized by an excess in the $15 \mu \mathrm{m} / 7 \mu \mathrm{m}$ colour (e.g. Roussel et al. 2001a; Dale et al. 2001; Förster Schreiber, Roussel, \& Sauvage, in prep.). From radiative transfer modeling of NGC 1808's 3-200 $\mu$ m spectrum, Siebenmorgen et al. (2001) concluded that the MIR range is dominated by PAHs and large dust grains (radii between 100 and $2400 \AA$ ) with negligible contribution by very small graphites and silicates (radii of 5-80 $\AA$ ). The key feature of their models is the inclusion of hot spots where large grains are heated locally by massive stars to higher temperatures giving rise to the $25 \mu \mathrm{m}$ emission and gradually contributing less towards shorter wavelengths where PAHs take over as main emitters.

We note that the morphology of the [Ar II] $6.99 \mu \mathrm{m}$ emission for M 82 and NGC 1808, especially the off-nucleus peak position, reflects the spatial distribution of the $\mathrm{H}_{\text {II }}$ regions and not variations in the excitation state of the ionized gas whereby, e.g., [Ar III] emission would become important at the expense of [Ar II]. As discussed in Sect. 3.2.1, the $\mathrm{H}_{\text {II }}$ regions in M 82 are concentrated in a circumnuclear ring-like structure at radius of $\approx 85$ pc. Direct tracers such as near-infrared to radio $\mathrm{H}$ recombination lines, MIR Ne, Ar, and S fine-structure lines, the $\mathrm{He}_{\text {I }} 2.06 \mu \mathrm{m}$ recombination line, and radio thermal continuum all show a similar distribution characterized by peaks flanking the nucleus, with the southwestern complex being the most intense, and little emission at the nucleus (e.g. Satyapal et al. 1995; Achtermann \& Lacy 1995; Seaquist et al. 1996; Förster Schreiber et al. 2001). In particular, the [Ar III] $8.99 \mu \mathrm{m}$ map of Achtermann \& Lacy (1995) tracing the $\geq 28 \mathrm{eV}$ radiation field clearly shows this bilobal distribution and relative lack of emission at the nucleus. Less information is available for NGC 1808 but $\mathrm{Br} \gamma$ imaging clearly reveals the $\mathrm{H}$ II region complexes to lie predominantly to the east and southeast of the nucleus, thus indicating a circumnuclear location for the starburst (Krabbe et al. 1994; Kotilainen et al. 1996).

Extinction effects could partly account for differences in the PAH, VSG, and [Ar II] $6.99 \mu \mathrm{m}$ emission on small spatial scales (comparable to the resolution elements in our ISOCAM data) but are not likely to affect the relative concentrations. The highest levels of obscuration are measured in the near surroundings of the MIR peaks in all three galaxies and generally decrease at larger distances (e.g. Larkin et al. 1994; Engelbracht et al. 1998; Krabbe et al. 1994). Depending on the extinction law applicable in the $3-10 \mu \mathrm{m}$ region (Sect. 5.1 below), such a radial gradient in extinction would either affect very little the relative global distributions of the tracers discussed here, or suppress more severely the shorter wavelength emission in 

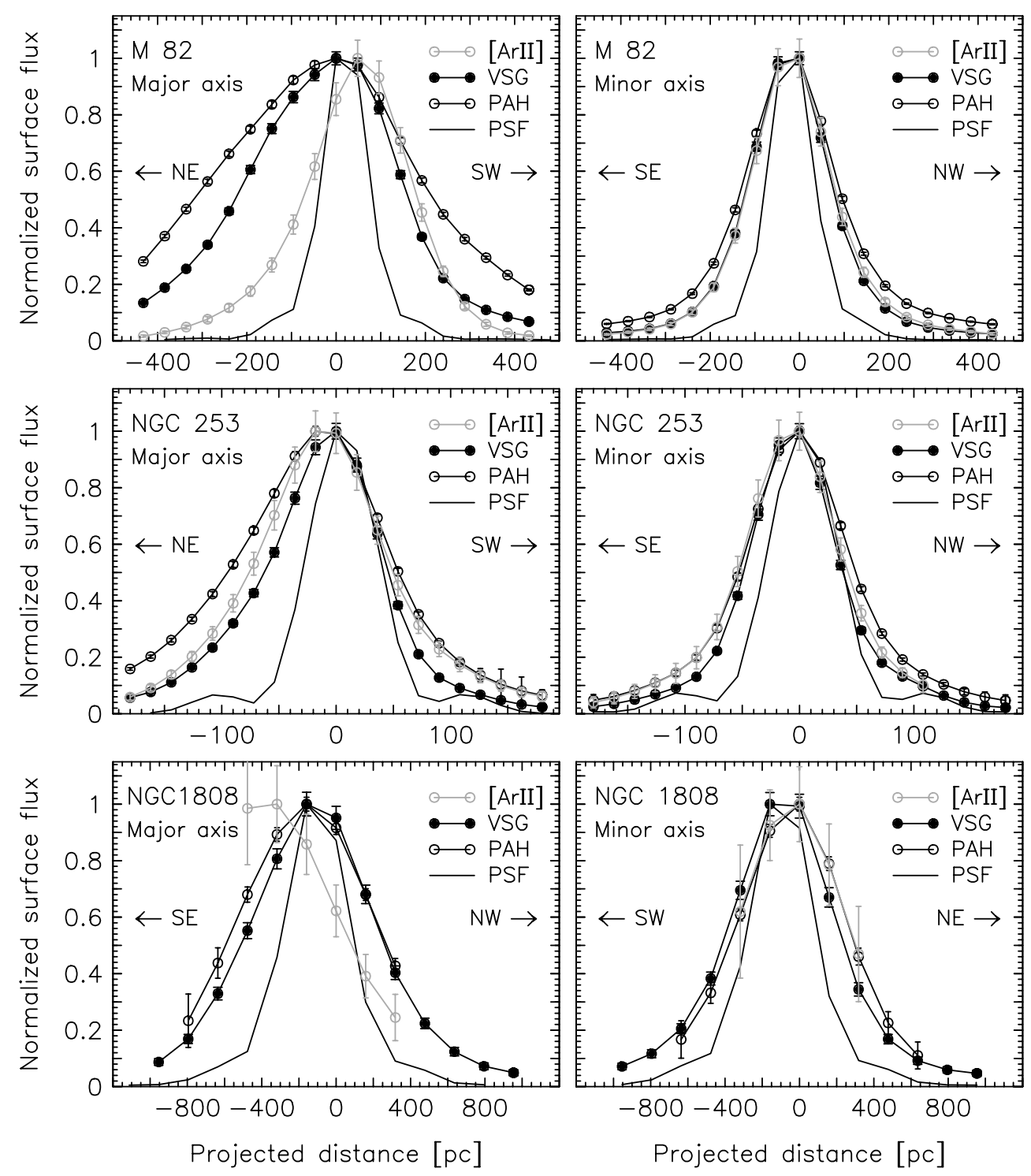

Fig. 9. Major and minor axis light profiles for M 82, NGC 253, and NGC 1808. The curves represent the variations of the integrated flux per unit surface area as a function of projected distance from the nucleus for the [Ar II] $6.99 \mu \mathrm{m}$ line (grey lines and open circles), the 13.5-15.0 $\mu \mathrm{m}$ VSG-dominated continuum (black lines and filled circles), and the 6.0-9.0 $\mu \mathrm{m}$ PAH-dominated region (black lines and open circles). The width of the synthetic slits along each axis is 3 pixels for M 82 and NGC 1808 and 6 pixels for NGC 253 (corresponding to 144, 477, and $108 \mathrm{pc}$, respectively). The curves are normalized to the maximum values which are for M 82, NGC 253, and NGC 1808, respectively: 0.26, 0.36 , and $0.0036 \mathrm{mJy} \mathrm{pc}^{-2}$ for the "PAH" major axis profiles, $0.28,0.38$, and $0.0035 \mathrm{mJy} \mathrm{pc}^{-2}$ for the "PAH" minor axis profiles, $0.53,1.20$, and $0.0044 \mathrm{mJy} \mathrm{pc}^{-2}$ for the "VSG" major axis profiles, $0.57,1.27$, and $0.0044 \mathrm{mJy} \mathrm{pc}^{-2}$ for the "VSG" minor axis profiles, $6.32,7.87$, and $0.035 \times 10^{-19} \mathrm{~W} \mathrm{~m}^{-2} \mathrm{pc}^{-2}$ for the [Ar II] major axis profiles, and 5.77, 8.30, and $0.024 \times 10^{-19} \mathrm{~W} \mathrm{~m}^{-2} \mathrm{pc}^{-2}$ for the [Ar II] minor axis profiles. The PSF profiles for the spatially-smoothed data cubes are shown as well (simple black lines; see Sect. 2).

the central regions, which is inconsistent with the overall trends for the $15 \mu \mathrm{m}$ continuum and [Ar II] $6.99 \mu \mathrm{m}$ line.

The nature of the short-wavelength continuum in normal and pure starburst galaxies is still debated. It is much weaker than in systems hosting an active galactic nucleus (AGN) where it is attributed to hot dust $(150-1700 \mathrm{~K})$ close to the AGN (Genzel \& Cesarsky 2000 and references therein). In non-AGN galaxies, the existence of 3-5 $\mu \mathrm{m}$ featureless continuum from small transiently heated dust grains underlying the PAH features has been proposed (e.g. Helou et al. 2000). On the other hand, the PAH features are best fitted with Lorentzian profiles whose wide wings can account for the apparent continuum pedestal (e.g. Boulanger et al. 1998b). Our narrow-band $5.5 \mu \mathrm{m}$ continuum map of M 82 provides only tentative evidence of a more extended distribution than for the $15 \mu \mathrm{m}$ continuum emission which would suggest a closer association of the emitting particles with PAHs than VSGs (see also Mattila et al. 1999).

\section{PAH and continuum emission: Relations to the ISM conditions and star-forming activity}

The two most striking aspects seen in the spectra presented in Fig. 3 are their remarkable similarity in the $\lambda=5-11 \mu \mathrm{m}$ regime dominated by $\mathrm{PAH}$ emission, and the spread in relative 
intensity of the longer wavelength emission attributed to VSGs. In this section, we focus on these two spectral components after discussing extinction effects at MIR wavelengths.

\subsection{The effects of extinction at mid-infrared wavelengths}

Interstellar extinction can significantly affect the MIR spectral energy distribution (SED) in obscured sources. In this regard, Rigopoulou et al. (1999) noted the gradual suppression of the PAH $8.6 \mu \mathrm{m}$ feature in progressively obscured starbursts and ULIRGs. They further proposed that variations in the PAH $6.2 \mu \mathrm{m} / 7.7 \mu \mathrm{m}$ ratio are dominated by extinction effects on the basis of the trend of decreasing ratio with increasing extinction observed in a subset of their sample for which reliable extinction determinations were available.

Another important consideration concerns the trough centered near $9.7 \mu \mathrm{m}$ traditionally attributed to absorption by interstellar silicate dust grains. Recent studies have cast doubt on the reliability of extinction estimates based on the observed feature depth. Sturm et al. (2000) discussed this issue in detail based on SWS data of M 82 and NGC 253, from a comparison with the spectrum of the Galactic reflection nebula NGC 7023 and from considerations of the relative optical depths expected for silicate absorption near 9.7 and $18 \mu \mathrm{m}$. In particular, they showed that the M 82 SWS spectrum, including the dip at $9.7 \mu \mathrm{m}$, can be well reproduced by a combination of the NGC 7023 spectrum and a power law rising from $8.5 \mu \mathrm{m}$ longwards without invoking large extinctions. More generally, the overall spectral invariance in the $5-11 \mu \mathrm{m}$ region among a variety of Galactic and extragalactic sources which are known or expected to cover a large range in extinction seems to argue in favour of the $9.7 \mu \mathrm{m}$ "absorption feature" being predominantly due to the gap between the main PAH emission complexes (e.g. Helou et al. 2000).

These suggestions raise the important question of how such an interpretation can be reconciled with the large and variable extinction in M 82, NGC 253, and NGC 1808 determined by alternative methods based, for instance, on the relative intensities of $\mathrm{H}$ recombination lines. To address this issue, we explored quantitatively the effects of extinction at MIR wavelengths by applying a range of extinction to a representative template spectrum constructed in a similar way as Sturm et al. (2000). We added to a PDR component (from the ISOCAM data of NGC 7023) a power law $f_{v} \propto(\lambda-8.5)^{\alpha}$ which represents well the VSG emission seen in our data. We adopted an index $\alpha=1.5$ and scaled the two components so as to obtain resulting spectra similar to that of M 82. For the purpose of illustrating the effects of different levels of extinction, the choice of these parameters is irrelevant.

We considered two representative geometries: a uniform foreground dust screen and a homogeneous mixture of dust and sources. For these models, the observed intensity $I(\lambda)$ of the emerging radiation is proportional to the total intrinsic intensity of the sources $I_{0}(\lambda)$ by the factors $\mathrm{e}^{-\tau_{\lambda}}$ and $\left(1-\mathrm{e}^{-\tau_{\lambda}}\right) / \tau_{\lambda}$, respectively, where $\tau_{\lambda}$ is the optical depth related to the extinction in magnitude via $A_{\lambda}=1.086 \tau_{\lambda}$. We adopted the extinction law proposed by Draine (1989) and investigated the effects of deviations from this law in the 3-10 $\mu \mathrm{m}$ region as found towards the Galactic Center (Lutz 1999; hereafter simply "GC law"). Such deviations are consistent with the H recombination line spectrum observed with the SWS in M 82 (Förster Schreiber et al. 2001). We varied the level of extinction specified in visual magnitudes $A_{V}$ for the mixed model in the range $A_{V}^{\mathrm{MIX}}=0-700$ mag. For a meaningful comparison, we varied $A_{V}$ for uniform foreground obscuration in the range $A_{V}^{\mathrm{UFS}}=0$ $50 \mathrm{mag}$, which results in the same attenuation at $10 \mu \mathrm{m}$ as the range considered for the mixed model. We normalized each extincted model spectrum to the integrated flux between 6.0 and $6.6 \mu \mathrm{m}$, as for the spectra of Fig. 3 .

Figure 10 presents our simulations. The plots outline the distinct behaviours for the two geometries considered with increasing $A_{V}$. They especially emphasize the fact that for the mixed model, differential extinction effects over the spectrum rapidly reach an asymptotic regime where large variations in $A_{V}$ become less and less perceptible as $A_{V}$ increases. In addition, except for the most extreme cases $\left(A_{V}>20 \mathrm{mag}\right)$, the choice of dust and source geometry has little impact. This is because the absolute levels of extinction are fairly small and the wavelength dependence of extinction is relatively weak; the wavelength range covered is thus too limited to probe appreciably different optical depths which could allow the discrimination between different dust and sources distributions.

For the Draine extinction law, the effects are largest between 8 and $13 \mu \mathrm{m}$. Our models illustrate well how the PAH 8.6 and $11.3 \mu \mathrm{m}$ features are substantially suppressed as extinction increases, indicating that their ratio with the PAH features at 6.2 and $7.7 \mu \mathrm{m}$ can be much affected by obscuration. Moreover, for a continuum level defined over the limited 8-13 $\mu \mathrm{m}$ interval, relatively small optical depths would be inferred even if the extinction is in fact large. Assuming the GC law at 3-10 $\mu \mathrm{m}$ results in a remarkably different behaviour. The extinction effects are significantly smaller in the $8-13 \mu \mathrm{m}$ region and the suppression of the PAH 8.6 and $11.3 \mu \mathrm{m}$ features is much less important. The largest effects are observed in the relative level between the short and long wavelength regions. This is a consequence of the much flatter GC law between 3 and $10 \mu \mathrm{m}$.

Our simulations indicate that dust obscuration adds a significant degree of degeneracy in the interpretation of the observed MIR emission, complicating the determination of intrinsic properties. Depending on the extinction law used, the shape of the PAH 8.6 and $11.3 \mu \mathrm{m}$ features as well as their flux ratio with the PAH 6.2 and $7.7 \mu \mathrm{m}$ features vary considerably. Therefore, extinction estimates based on diagnostics involving the PAH 8.6 or $11.3 \mu \mathrm{m}$ feature may be strongly biased by the choice of extinction law. Furthermore, part of the spread in intensity observed in the long-wavelength continuum relative to the shorter wavelength emission could be attributed to varying extinction levels (see Fig. 3 and Sect. 5.2 below).

The interpretation of the $9.7 \mu \mathrm{m}$ trough as largely due to the gap between the flanking PAH complexes appears to hold over a wide range of extinction, especially for the GC extinction law. The near-invariance of the 5-11 $\mu \mathrm{m}$ spectrum is therefore not inconsistent with large and/or variable obscuration among and within galaxies. We illustrate this with the case of M 82, 
for which a mixed model with $A_{V}=52$ mag best reproduces the observed $\mathrm{H}$ recombination lines from radio to optical wavelengths while the best-fit uniform foreground screen extinction of $A_{V}=5$ mag provides a much poorer fit (Förster Schreiber et al. 2001). We applied these two extinction models to the same template as for the simulations described above. The resulting spectra are plotted along with the observed spectrum of M 82 in the bottom panels of Fig. 10. We did not attempt to fine-tune the models by formal fitting. Given the uncertainties on the exact nature of the emitting particles, specific assumptions on model parameters are not well constrained and a simple empirical approach is sufficient for our purposes. The comparison shows that strictly from the point of view of the 5-16 $\mu \mathrm{m}$ range, both extinction models reproduce equally well the observed SED of M 82, thus demonstrating that a high extinction cannot be excluded from the overall shape of the MIR spectrum alone.

We wish to emphasize that we do not dismiss the possibility of silicate absorption around $9.7 \mu \mathrm{m}$ in general but merely want to point out the difficulties involved in the interpretation of the observed feature. The SEDs of moderately to highly obscured sources including ULIRGs often exhibit a strong dip near $9.7 \mu \mathrm{m}$ together with suppressed PAH 8.6 and $11.3 \mu \mathrm{m}$ features relative to those at 6.2 and $7.7 \mu \mathrm{m}$, consistent with the presence of silicate grains (e.g. Dudley \& Wynn-Williams 1997; Dudley 1999; Laurent et al. 2000; Le Floc'h et al. 2002). Given the near invariance of the PAH spectrum in a wide range of environments, it should be possible to define an indicator measuring the differential extinction between the $9.7 \mu \mathrm{m}$ region and adjacent less affected intervals that quantifies the absolute extinction. One must however be aware of the importance of sufficient wavelength coverage and resolution to assess properly the impact of the PAH complexes which can make the silicate absorption look artificially deep, of the dependence of the inferred $A_{V}$ on the assumed extinction law and spectral intervals used to measure the feature depth, and of the limited sensitivity of this diagnostic leading to rather large uncertainties in the derived $A_{V}$. The upcoming launch of SIRTF in 2003 will provide $5-40 \mu \mathrm{m}$ spectroscopy with an increase in sensitivity by two orders of magnitude and will help us address this issue by enabling a better sampling of the continuum emission as well as both the 9.7 and $18 \mu \mathrm{m}$ silicate bands.

\subsection{The $\lambda \geq 11 \mu m$ continuum emission}

The $\lambda \gtrsim 11 \mu \mathrm{m}$ continuum emission in our data exhibits a large spread in intensity relative to the shorter wavelength emission. Similar variations have been seen in normal spirals and starburst galaxies observed with ISO instruments and, based on evidence provided by Galactic sources, are generally interpreted in terms of the relative contribution of $\mathrm{H}$ II regions to the MIR emission (e.g. Laurent et al. 2000; Roussel et al. 2001a; Dale et al. 2001). A close link between the $15 \mu \mathrm{m} / 7 \mu \mathrm{m}$ colour as measured through the ISOCAM broad-band LW3 and LW2 filters $(\lambda=12-15 \mu \mathrm{m}$ and $5-8.5 \mu \mathrm{m}$, respectively) and the IRAS $25 \mu \mathrm{m} / 12 \mu \mathrm{m}$ colour has been emphasized by Dale et al. (2001) and Roussel et al. (2001a).
In order to assess quantitatively the relationship between the long-wavelength continuum properties and the $\mathrm{H}_{\text {II }}$ regions, we investigated the relationship between the $15 \mu \mathrm{m}$ narrowband continuum and the [ArII] $6.99 \mu \mathrm{m}$ line emission. The [Ar II] $6.99 \mu \mathrm{m}$ is the most direct probe of $\mathrm{H}_{\mathrm{II}}$ regions and the least contaminated by $\mathrm{PAH}$ emission available from our ISOCAM data sets. The $\mathrm{Ar}^{0}$ ionization potential of $15.8 \mathrm{eV}$ is close to that of $\mathrm{H}(13.6 \mathrm{eV})$. Argon being a noble element, it is, like neon, not expected to be significantly depleted onto dust grains; while the argon gas-phase abundance may increase with time as a result of star formation activity and differ among galaxies, it is not likely to vary significantly over scales of $\sim 100-1000 \mathrm{pc}$ as covered by the starburst regions in our sample. For the relatively low nebular excitation in all three sources (Fig. 4; see also Sturm et al. 2000; Förster Schreiber et al. 2001), argon is mostly singly-ionized so that the [Ar II] $6.99 \mu \mathrm{m}$ emission should trace the HiI regions in much the same way as $\mathrm{H}$ recombination lines and its luminosity, scale with the radiation field intensity of the young stars. In addition, since the gas-phase abundances of the galaxies are solar within a factor of a few (e.g. Webster \& Smith 1983; Forbes et al. 1992; Förster Schreiber et al. 2001), the proportionality factor should be roughly similar.

Figure 11 presents the $15 \mu \mathrm{m}$ continuum versus [Ar II] $6.99 \mu \mathrm{m}$ luminosities for our galaxies, normalized to unit projected surface area $\left(\Sigma_{15 \mu \mathrm{m}}\right.$ and $\left.\Sigma_{[\mathrm{ArII}]}\right)$. The data are shown for the selected regions as well as for the individual resolution elements enclosed within the radii defining the outer limit of the disk regions in M 82 and NGC 253. Resolution elements with measurements at $<3 \sigma$ were excluded. The data points follow a well defined distribution, with a remarkable overlap for the different galaxies. Least-squares fitting to the resolution elements' data accounting for the individual formal uncertainties yields the relation

$\log \left(\Sigma_{15 \mu \mathrm{m}}\right)=1.01 \log \left(\Sigma_{[\mathrm{ArII}]}\right)+0.807$

with dispersion around the fit of 0.203 dex. The extinction paths in the diagram of Fig. 11 are nearly parallel to the locus formed by the data points; however, implausibly large differential extinctions would be required in order to account entirely for the observed distribution.

The essentially linear proportionality we find between $\Sigma_{15 \mu \mathrm{m}}$ and $\Sigma_{[\mathrm{ArII}]}$ indicates that the $15 \mu \mathrm{m}$ emission provides a good quantitative indicator of the star-forming activity in starburst environments, to within uncertainties determined by the dispersion of the data and by extinction (a factor of 2.5 for an $A_{V}=50 \mathrm{mag}$ assuming purely foreground extinction). Roussel et al. (2001b) reached a similar conclusion for more quiescent spiral disks where the $12-15 \mu \mathrm{m}$ emission scales linearly with the $\mathrm{H} \alpha$ line flux. Part of the scatter in Fig. 11 may result from variations in the physical conditions and exact composition of the gas and dust within and between the galaxies. More important factors, however, are variations in relative spatial distribution of the emission and possibly of the excitation state of the gas.

The observed relationship holds remarkably well in view of the differences in morphology of the $15 \mu \mathrm{m}$ continuum and [Ar II] $6.99 \mu \mathrm{m}$ line emission as seen in Figs. 5-7, and 9. 

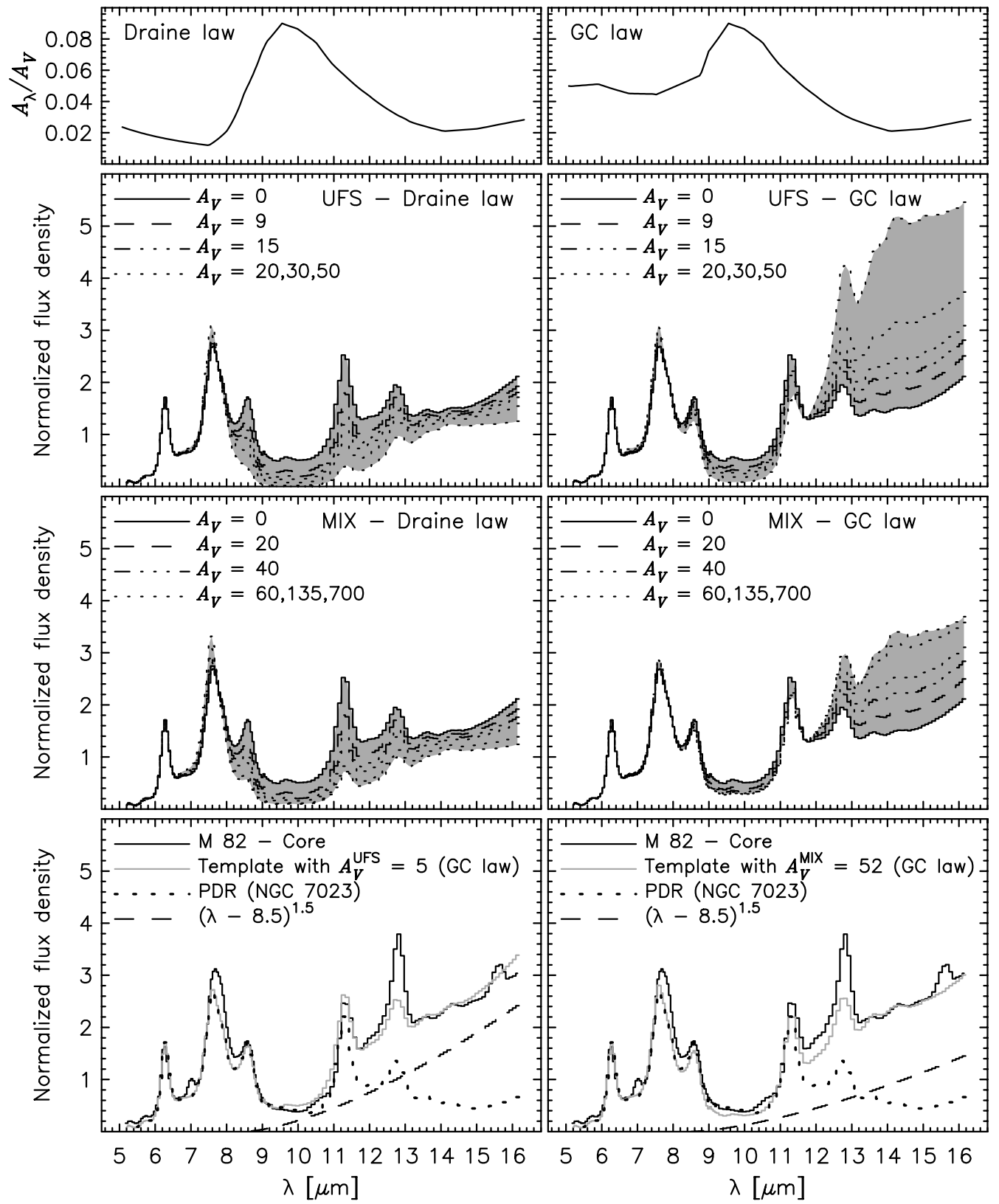

Fig. 10. Extinction effects at MIR wavelengths. The top panels show the extinction law from Draine (1989), and the modifications to this law at $\lambda \leq 10 \mu \mathrm{m}$ appropriate for the Galactic Center line of sight (Lutz 1999). The middle panels show the variations of the SED of a template spectrum affected by various levels of extinction, for different dust and source geometries and extinction laws, as labeled in each plot. The template combines the spectrum of the Galactic PDR NGC 7023 and a power-law spectrum (shown individually in the bottom panels by the dashed and dotted lines, respectively). "UFS" and "MIX" stand for uniform foreground screen and mixed model. The grey shading outlines the range of variations for $A_{V}^{\mathrm{UFS}}=0-50 \mathrm{mag}$ and $A_{V}^{\mathrm{MIX}}=0-700 \mathrm{mag}$. The various curves show the results for selected extinction levels, corresponding to similar attenuation factors at $10 \mu \mathrm{m}$ between the two extinction models (labeled in each plot). The bottom panels show nonformal fits (grey solid line) to the spectrum of the starburst core of M 82 (black solid line), using the template PDR + power-law spectrum, attenuated by the best-fit extinction for each geometry assuming the GC extinction law and derived from radio to optical $\mathrm{H}$ recombination line measurements (see Sect. 5.1). All spectra are normalized to the total flux between 6.0 and $6.6 \mu \mathrm{m}$, except for the power-law.

The correlation extends however over two to three orders of magnitude, much larger than the dispersion of a factor of 1.6. Undoubtedly, the relative spatial variations between both tracers contribute significantly to the scatter. Within the starburst cores of M 82 and NGC 253, where the steeply rising SEDs at $\lambda \gtrsim 11 \mu \mathrm{m}$ are dominated by VSG emission, the differences may be attributed to different ranges in exciting photon energies for VSGs and [ArII] $6.99 \mu \mathrm{m}$ line as well as to extinction effects (Sect. 4). For NGC 1808, the spatial distributions differ probably because a different dust/particles population produces the flat long-wavelength continuum (Sect. 4). Nevertheless, the data for NGC 1808 and for the disk regions of M 82 and NGC 253, characterized by flat $\gtrsim 11 \mu \mathrm{m}$ SEDs, are well described by Eq. (1). 
Variations of the excitation state of the photoionized nebulae may influence the $\Sigma_{15 \mu \mathrm{m}}$ versus $\Sigma_{\text {[Ar II] }}$ relationship as well. In particular, the [Ar III] $8.99 \mu \mathrm{m}$ line is fairly strong in the $I S O$ SWS spectrum of M 82 (Fig. 4) but the [Ar III] $8.99 \mu \mathrm{m} /[$ Ar II] $6.99 \mu \mathrm{m}$ ratio of 0.18 ( 0.26 after extinction correction) is low and abundance estimates indicate that $\sim 25 \%$ only of the argon is doubly ionized (Förster Schreiber et al. 2001). As emphasized in Sect. 4, the spatial distributions of various MIR fine-structure lines and $\operatorname{Br} \alpha$ are similar and suggest a roughly constant excitation state of the $\mathrm{H}_{\text {II }}$ regions across M 82 (Achtermann \& Lacy 1995). This is further confirmed by the nearly uniform $\mathrm{He}_{\mathrm{I}} 2.06 \mu \mathrm{m} / \mathrm{Br} \gamma$ ratio in the central $260 \times 160$ pc which corresponds closely to the excitation derived from the SWS [Ne III] $15.56 \mu \mathrm{m} /[\mathrm{Ne}$ II $] 12.81 \mu \mathrm{m}$, [Ar III] $8.99 \mu \mathrm{m} /\left[\right.$ Ar II] $6.99 \mu \mathrm{m}$, and [S IV] $10.5 \mu \mathrm{m} /\left[\mathrm{S}_{\mathrm{III}}\right]$ $18.7 \mu \mathrm{m}$ ratios within larger apertures up to $430 \times 225 \mathrm{pc}$ (Förster Schreiber et al. 2001). Similar arguments are more difficult for NGC 253 and NGC 1808 because of the lack of relevant data. However, the [Ar III] $8.99 \mu \mathrm{m} /[$ Ar II] $6.99 \mu \mathrm{m} \approx 0.03$ measured from the SWS spectrum of NGC 253 is substantially lower than for M 82 , as is the [Ne III] $15.56 \mu \mathrm{m} /[\mathrm{Ne}$ II] $12.81 \mu \mathrm{m}$ ratio (0.06 compared to 0.18 ; Thornley et al. 2000 ; see also Giveon et al. 2002 for the relationship between these two MIR line ratios). For NGC 1808, we can only note from the ISOCAM spectra (Fig. 3) that the weakness of the $15.7 \mu \mathrm{m}$ feature and the non-detection of [Ar III] $8.99 \mu \mathrm{m}$ indicate low excitation supporting that argon mostly is singly ionized. Summarizing, the excitation state of the gas might cause for M 82 a general but small offset to the left in the relationship of Fig. 11 compared to the other galaxies because of the larger fraction of $\mathrm{Ar}^{++}$while spatial variations are not likely to introduce scatter larger than this offset. These effects are expected to be smaller for both NGC 253 and NGC 1808.

The relationship between the $15 \mu \mathrm{m}$ continuum and star formation intensity is supported by the analysis of a larger sample including spiral and starburst galaxies that will be presented in a forthcoming paper (Förster Schreiber, Roussel, \& Sauvage, in preparation). In this paper, based on the previous work of Roussel et al. (2001b) for spiral disks, we find that the $15 \mu \mathrm{m}$ continuum as well as the $7 \mu \mathrm{m}$ PAH-dominated emission correlate well with the star formation rate over nearly 7 orders of magnitude, with the interesting distinction of two regimes that correspond to quiescent star formation in disks and more intense activity in circumnuclear regions and starbursts.

\subsection{The $\lambda=5-11 \mu \mathrm{m}$ emission}

The near invariance of the $5-11 \mu \mathrm{m}$ spectrum seen in our data of M 82, NGC 253, and NGC 1808 has been noted in a number of other studies of different types of galaxies powered mainly by star formation, as well as in a variety of Galactic sources (see Tielens et al. 1999 for a review; see also e.g. Boulanger et al. 1998a; Helou et al. 2000; Uchida et al. 2000). This indicates that the PAHs are very stable under a wide range of physical conditions despite their small sizes (typically $\sim 100$ atoms) and is taken as direct observational evidence for the stochastic nature of the emission processes involved. Furthermore, if different types of PAHs coexist, the near constancy in the 5$11 \mu \mathrm{m}$ region suggests that their relative abundances vary little. Finally, it has consequences on the interpretation of and extinction measurements from the $9.7 \mu \mathrm{m}$ silicate absorption feature because of the intrinsic gap between the main 6-9 $\mu \mathrm{m}$ and 11$13 \mu \mathrm{m}$ PAH complexes (Sect. 5.1).

Variations of the relative intensities of the PAH features do exist, however. We focus on the PAH $6.2 \mu \mathrm{m} / 7.7 \mu \mathrm{m}$ and PAH $8.6 \mu \mathrm{m} / 11.3 \mu \mathrm{m}$ ratios, maps of which were presented for M 82 and NGC 253 in Sect. 3.2. Of particular interest is also the enhancement of the PAH $11.3 \mu \mathrm{m}$ relative to the other features in NGC 1808 compared to M 82 and NGC 253, evident in Fig. 3. The variations of PAH ratios in our sample are comparable to those observed within and between Galactic sources of similar types (e.g. Cesarsky et al. 1996a; Boulanger et al. 1998a; Lu 1998; Crété et al. 1999; Uchida et al. 2000), along the disk of the spiral galaxy NGC 891 (Mattila et al. 1999), and among a sample of 15 starbursts and ULIRGs (Rigopoulou et al. 1999). A detailed discussion of $\mathrm{PAH}$ ratios is beyond the scope of this paper, but we briefly mention possible interpretations of the variations seen in our data in the light of some recent theoretical and empirical work.

Since the emission mechanism is stochastic in nature, $\mathrm{PAH}$ ratios are not directly related to the SED of the incident radiation (e.g. Boulanger et al. 1998a; Uchida et al. 2000). On the other hand, they may carry indirect information since PAHs exposed to hard and intense radiation fields can be ionized, lose $\mathrm{H}$ atoms, or be photodissociated (e.g. Léger et al. 1989; Allamandola et al. 1989; Schutte et al. 1993; Allamandola et al. 1999; Hudgins \& Allamandola 1999). In particular, the PAH $8.6 \mu \mathrm{m} / 11.3 \mu \mathrm{m}$ is believed to trace the fraction of singly ionized to neutral PAHs, presumably driven by the strength of the radiation field from the OB stars (see references above, and Joblin et al. 1996). Draine \& Li (2001) presented a thorough calculation of the expected PAH spectrum as a function of various parameters including $\mathrm{PAH}$ size, charging conditions, and starlight intensity. Their results indicate that the PAH $6.2 \mu \mathrm{m} / 7.7 \mu \mathrm{m}$ depends primarily on PAH size while the PAH $11.3 \mu \mathrm{m} / 7.7 \mu \mathrm{m}$ is mainly sensitive to the fraction of ionized versus neutral PAHs and only modestly to PAH size. The effects of radiation field become noticeable only at high intensities $\left(\gtrsim 10^{5}\right.$ the average local Galactic far-UV flux in Habing units) and for large PAHs with $\gtrsim 10^{2}$ carbon atoms. Mattila et al. (1999) suggested that PAH $6.2 \mu \mathrm{m} / 7.7 \mu \mathrm{m}$ variations may be due to differences in average temperature of the PAHs during their temperature spikes, related to PAH size or mean exciting UV photon energies. Alternatively, they could be attributed to broadening of the $7.7 \mu \mathrm{m}$ feature at low radiation field energy densities (Uchida et al. 2000); this would reduce the PAH $7.7 \mu \mathrm{m}$ flux and increase the continuum level within our fixed bandpasses. In addition to intrinsic variations, extinction can significantly alter the shape and relative intensities of the PAH features as shown in Sect. 5.1.

Of our sample galaxies, M 82 offers the most interesting case for interpretation. The overall bilobal structure in the maps of PAH ratios and $\mathrm{CO}$ gas distribution (Fig. 8) might result from the different composition and physical processes that the 


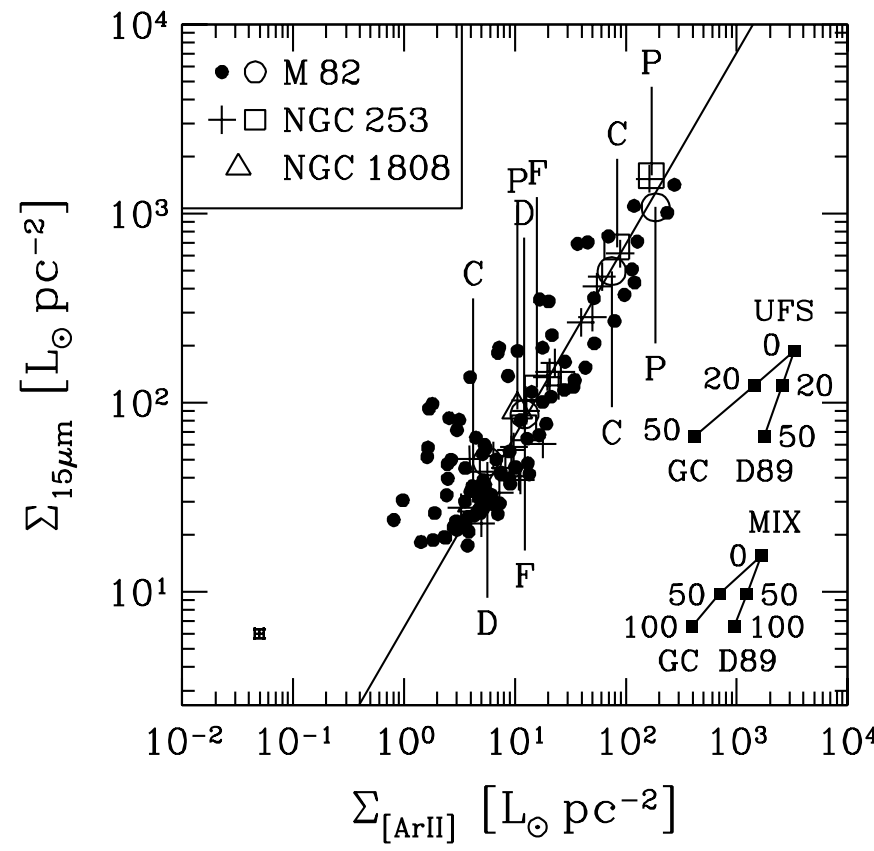

Fig. 11. Relationship between MIR tracers of H II regions in M 82, NGC 253, and NGC 1808. The diagram shows the variations of the $15 \mu \mathrm{m}$ continuum luminosity as a function of the [Ar II] $6.99 \mu \mathrm{m}$ line luminosity normalized per unit projected area. Data are plotted for selected regions (open symbols labeled "F" for the ISOCAM field of view, "D" for the disk regions, "C" for the starburst core, and "P" for the MIR peak). The filled circles and crosses show the values for individual resolution elements for M 82 and NGC 253, respectively, within radii of $30^{\prime \prime}$ and $15^{\prime \prime}$ (outer disk annulus; Table 2); measurements at $<3 \sigma$ are excluded (4 points for M 82, 11 for NGC 253). Typical formal uncertainties are indicated by the error bar at the bottom left and are smaller than the symbol sizes; they are $5 \%$ for $\Sigma_{15 \mu \mathrm{m}}$ and $10 \%$ for $\Sigma_{[\mathrm{Ar} I \mathrm{II}}$ (the median and average differ by $<1.5 \%$ ). The straight line is a least-squares fit to the resolution elements' data accounting for the individual uncertainties. The effects of extinction are shown in the right part of the plot for a uniform foreground screen and a mixed model ("UFS" and "MIX"), for the extinction law of Draine (1989, "D89") and with modifications at 3-10 $\mu \mathrm{m}$ as found towards the Galactic Center (Lutz 1999, "GC"). Selected values of extinction in visual magnitudes $A_{V}$ are labeled.

emitting PAHs undergo when exposed to the varying conditions across M 82, from the starburst core to the more quiescent disk via the transition regions marked by the molecular gas ring. The maxima in PAH $8.6 \mu \mathrm{m} / 11.3 \mu \mathrm{m}$ ratio lie at smaller radii than the minima in PAH $6.2 \mu \mathrm{m} / 7.7 \mu \mathrm{m}$ ratio, possibly indicating a higher degree of PAH ionization within the most intense starburst sites at the inner edge of the molecular ring; this is particularly striking southwest of the nucleus where the peak PAH $8.6 \mu \mathrm{m} / 11.3 \mu \mathrm{m}$ coincides very well with the location of the most prominent $\mathrm{H}$ II region complexes (Sect. 3.2.1). The variations of PAH $6.2 \mu \mathrm{m} / 7.7 \mu \mathrm{m}$ ratio could reflect differences in the PAH size distribution, combined with extinction effects, where larger PAHs can better form and survive in denser, more shielded environments associated with molecular gas concentrations. In NGC 253, the peak PAH $8.6 \mu \mathrm{m} / 11.3 \mu \mathrm{m}$ ratio at the nucleus where the starburst is mainly occurring could also be due to a larger fraction of ionized PAHs. Finally, the enhanced PAH $11.3 \mu$ m feature in NGC 1808 compared to M 82 and NGC 253 could be explained by a more neutral mixture of PAHs and a more diffuse radiation field.

The relative intensity of PAH and continuum emission can also hold information on the physical environment within astronomical sources. In particular, the ratio of the peak intensity of the PAH $7.7 \mu \mathrm{m}$ to the underlying continuum, hereafter PAH 7.7 $\mu \mathrm{m} \mathrm{L/C}$ ratio, has been shown to constitute a powerful discriminator between star formation activity or an AGN as the main source of the bulk of infrared luminosity (Rigopoulou et al. 1999; see also Genzel et al. 1998; Laurent et al. 2000; Tran et al. 2001). Starburst-dominated objects are characterized by ratios $\gtrsim 1$ while AGN-dominated ones have ratios $\lesssim 1$. We compared measurements of the PAH $7.7 \mu \mathrm{m} \mathrm{L} / \mathrm{C}$ ratio from our data of M 82, NGC 253, and NGC 1808 with those obtained by Rigopoulou et al. (1999) for a large sample including starbursts, AGNs, and ULIRGs. Our motivation was to assess how much this indicator depends on the source luminosity at the faint end and compare the spread in global ratios among different galaxies with the spatial variations within individual galaxies.

Figure 12 shows the PAH $7.7 \mu \mathrm{m} \mathrm{L} / \mathrm{C}$ ratio versus peak PAH 7.7 $\mu \mathrm{m}$ luminosity for selected regions of our galaxies, for individual resolution elements of M 82 and NGC 253 (within the radii defining the outer limit of the disk regions in Table 2), and for the Rigopoulou et al. (1999) sample. We measured the PAH $7.7 \mu \mathrm{m}$ peak intensity and underlying continuum according to the definitions of Rigopoulou et al. We note that with these definitions, the continuum might be underestimated in highly obscured sources as a result of extinction affecting notably the $11 \mu \mathrm{m}$ region (as also discussed by Laurent et al. 2000). We chose the PAH $7.7 \mu \mathrm{m}$ peak luminosity as other characteristic property, taken as an approximate indicator of the total infrared luminosity. Although the PAH to infrared luminosity ratio can vary by up to factors of several in different environments (Rigopoulou et al. 1999), our assumption has little consequences on the interpretation of Fig. 12 since the data span a range in luminosity extending over more than six orders of magnitude 4 .

Our data of M 82, NGC 253, and NGC 1808 extend very well the trend defined by the global properties of pure starburst galaxies and ULIRGs, populating the PAH $7.7 \mu \mathrm{m} \mathrm{L} / \mathrm{C}$ $\gtrsim 1$ region down to luminosities about an order of magnitude lower. The ratios in all three galaxies lie well above the starburst-AGN separation at a ratio of unity and form a tighter distribution. The average and $1 \sigma$ dispersion for the individual resolution elements in M 82 and NGC 253 are $5.0 \pm 0.9$ compared to $3.7 \pm 2.5$ for the pure starbursts of Rigopoulou et al. and $2.8 \pm 1.0$ for their ULIRGs (excluding those with ratios $\leq 1$ or with only limits on the measurements). It is also

${ }^{4}$ Additional though indirect support for our assumption is provided by the relationship between the $7 \mu \mathrm{m}$ flux measured through the ISOCAM LW2 filter (5-8.5 $\mu \mathrm{m})$ and the far-infrared (FIR, 43$123 \mu \mathrm{m})$ flux obtained by Roussel et al. (2001b) for galactic disks, with $F_{7 \mu \mathrm{m}} \propto\left(F_{\mathrm{FIR}}\right)^{1.2}$. Furthermore, Dale et al. (2001) derived a typical value of $\approx 15 \%$ for the $5-20 \mu \mathrm{m}$ to FIR luminosity ratio in normal galaxies while Charmandaris et al. (2002) found this ratio to be $\sim 5 \%$ for three ULIRGs. 


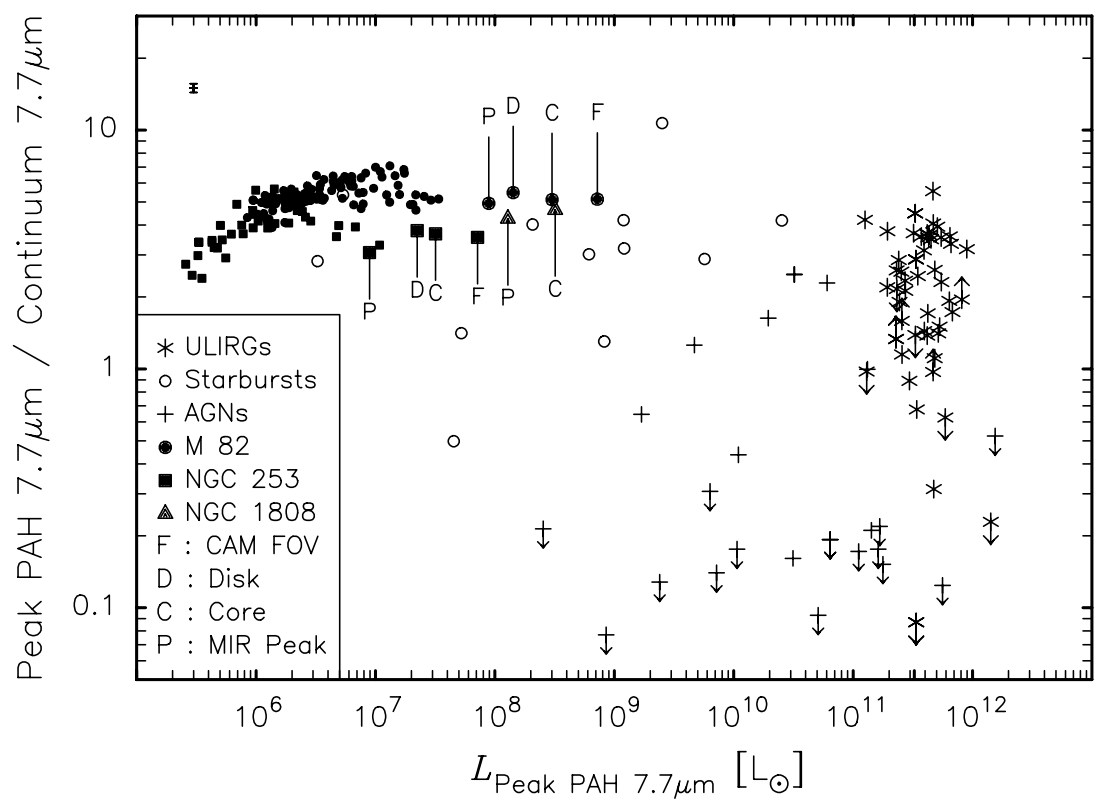

Fig. 12. Diagnostic diagram for dominant starburst versus AGN activity. The data for selected regions in M 82, NGC 253, and NGC 1808 as well as individual resolution elements for M 82 and NGC 253 (large labeled and small unlabeled filled symbols, respectively; see inset) are compared to the global properties for the samples of starburst galaxies, AGNs, and ULIRGs of Rigopoulou et al. (1999; open circles, crosses, and stars). For M 82 and NGC 253, resolution elements within radii of $30^{\prime \prime}$ and 15" (outer disk annulus; Table 2) are plotted, and typical formal uncertainties are indicated by the error bar at the top left: $<1 \%$ for $L_{\mathrm{Peak}} \mathrm{PAH} 7.7 \mu \mathrm{m}$ and $4 \%$ for PAH $7.7 \mu \mathrm{m} \mathrm{L/C}$ ratio (uncertainties are comparable to or smaller than the symbol sizes; average and median values differ by $<1 \%$ ).

interesting to note that the starburst trend holds for regions on spatial scales ranging from $\approx 60 \mathrm{pc}$ for the smallest individual regions in NGC 253 up to several kiloparsecs for the largest starbursts and ULIRGs (see the near-infrared images of Rigopoulou et al. 1999).

\section{Summary and conclusions}

We have presented ISOCAM $\lambda=5.0-16.5 \mu \mathrm{m}$ spectrophotometric imaging of the starburst galaxies M 82, NGC 253, and NGC 1808. The spectrum of all three objects, down to the smallest scales of $\sim 100 \mathrm{pc}$ accessible from our data, exhibit similar characteristics including prominent PAH bands, a featureless continuum most obvious at $\lambda \gtrsim 11 \mu \mathrm{m}$, and a trough in the $10 \mu \mathrm{m}$ region. We securely identified the main emission features detected in the $R \sim 40$ ISOCAM data of M 82 and NGC 253 based on their $R \sim 500-1000$ SWS spectra. The comparison emphasizes the caution that should be exercised when interpreting low resolution data because of potential blends or misidentifications between emission lines from ionized gas and PAH features originating in PDRs, a notable example being [Ne II] $12.81 \mu \mathrm{m}$ and PAH $12.7 \mu \mathrm{m}$. Using a simple model combining a template PDR spectrum and a powerlaw $f_{v} \propto(\lambda-8.5)^{1.5}$, we constructed a representative starburst SED and explored the effects of extinction at MIR wavelengths. Our simulations illustrate the importance of the assumed extinction law (e.g. the widely used Draine 1989 law versus the Galactic Center law of Lutz 1999) and of the intrinsic PAH spectrum (especially the gap between the main 6-9 $\mu \mathrm{m}$ and 11$13 \mu \mathrm{m}$ complexes) in shaping the SED of astronomical sources. This complicates the interpretation of PAH ratios as well as extinction measurements relying on the silicate dust absorption at $9.7 \mu \mathrm{m}$ (see also Sturm et al. 2000).

As observed previously in a wide range of Galactic and extragalactic sources, the $5-11 \mu \mathrm{m}$ spectrum in our galaxies is nearly invariant. The relative PAH intensities exhibit nevertheless measureable and significant variations of $20 \%-100 \%$ which may be attributed to various, possibly interrelated effects including the intensity of the incident radiation field and the PAH size distribution, ionization, and dehydrogenation. In our sample, M 82 probably best illustrates variations of PAH ratios due to an increased fraction of ionized PAHs within the most intense starburst sites and, admittedly speculatively, perhaps also to differences in typical PAH sizes depending on the molecular gas concentrations. The PAH $7.7 \mu \mathrm{m} \mathrm{L} / \mathrm{C}$ ratio in all three galaxies clearly lies in the range observed for pure starburst systems and extends the trend reported previously by Rigopoulou et al. (1999) to lower luminosities.

In contrast, the $\lambda \gtrsim 11 \mu \mathrm{m}$ region varies most among our sample galaxies and the ISOCAM maps show a comparatively more compact $15 \mu \mathrm{m}$ continuum distribution relative to the PAH emission. Strong $15 \mu \mathrm{m}$ continuum in M 82 and NGC 253 indicates an important contribution by VSGs contrary to the case of NGC 1808 where the long-wavelength emission is much flatter presumably because of negligible contribution by VSGs. We find, however, that in all three galaxies the $15 \mu \mathrm{m}$ continuum and [Ar II] $6.99 \mu \mathrm{m}$ line fluxes satisfy a linear relationship. We infer from this that the $15 \mu \mathrm{m}$ continuum provides a good indicator of star formation activity in starbursts, complementing the similar results of Roussel et al. (2001b) for galactic disks. In a broader perspective, our galaxies fit well in the trend of increasing ISOCAM $15 \mu \mathrm{m} / 7 \mu \mathrm{m}$ ratios 
with higher levels of star formation activity found among normal disk galaxies and starburst-powered LIRGs/ULIRGs (e.g. Laurent et al. 2000; Roussel et al. 2001b; Dale et al. 2001).

The value of the ISOCAM spectrophotometric imaging of M 82, NGC 253, and NGC 1808 presented in this paper also lies in that it complements existing MIR data with maps of PAH features, fine-structure lines, and continuum components not previously imaged. The poorer angular resolution of ISOCAM compared to that achieved with large ground-based telescopes is compensated by the larger field of view revealing more of the large scale emission. In that respect, we stress the small size of the MIR source relative to the optical extent of all three galaxies: the ISOCAM maps cover the central $\approx 1.5 \mathrm{kpc}$ for M 82 , $0.6 \mathrm{kpc}$ for NGC 253, and $5 \mathrm{kpc}$ for NGC 1808 while the optical diameters are about $10 \mathrm{kpc}$ for M 82 and $20 \mathrm{kpc}$ for the other two. By measuring the flux density within the ISOCAM LW10 filter bandpass, equivalent to the IRAS $12 \mu \mathrm{m}$ band, we recover all of the IRAS $12 \mu \mathrm{m}$ emission in the entire field of view for M 82 and NGC 1808, and about $70 \%$ for NGC 253. This suggests that the total MIR emission is strongly dominated by the starburst sites in the nuclear regions while the more quiescent star formation taking place in the disk at larger radii does not contribute much. It will be interesting to see whether the MIR cameras on board SIRTF, to be launched in 2003, will confirm this result or discover faint diffuse emission, especially at shorter $(3-5 \mu \mathrm{m})$ wavelengths.

Acknowledgements. We are grateful to E. Sturm for making the SWS spectrum of NGC 253 available to us in electronic form. We warmly thank H. Roussel, A. Vogler, S. Madden, and especially D. Tran for many interesting discussions on various aspects of this work. We also wish to thank the referee, Dr. N. Bergvall, for his valuable comments that improved the quality of the paper. VC would like to acknowledge the partial support of JPL contract 960803.

\section{References}

Achtermann, J. M., \& Lacy, J. H. 1995, ApJ, 439, 163

Allamandola, L. J., Hudgins, D. M., \& Sandford, S. A. 1999, ApJ, 511, L115

Allamandola, L. J., Tielens, A. G. G. M., \& Barker, J. R. 1989, ApJS, 71,733

Anantharamaiah, K. R., \& Goss, W. M. 1996, ApJ, 466, L13

Biviano, A., Sauvage, M., \& Roman, P. 1997, ESA/CEA Technical Report, December 1997

Biviano, A., Altieri, B., Blommaert, J., et al. 1998a, The ISOCAM CVF Calibration Report, version 1.1, ESA/CAM IDT Vilspa Technical report

Biviano, A., Blommaert, J., Laurent, O., et al. 1998b, The ISOCAM Flat Field Calibration Report, version 1.1, ESA/CAM IDT Vilspa Technical report

Blain, A. W., Smail, I., Ivison, R. J., Kneib, J.-P., \& Frayer, D. T. 2002, Phys. Rep., in press [astro-ph/0202228)]

Blommaert, J., \& Cesarsky, D. 1998, ISOCAM Calibration Accuracies Document, ESA/CAM IDT Vilspa Technical report

Böker, T., Krabbe, A., \& Storey, J. W. V. 1998, ApJ, 498, L115

Boulanger, F., Prévot, M. L., \& Gry, C. 1994, A\&A, 284, 956

Boulanger, F., Beichman, C., Désert, F. X., et al. 1988, ApJ, 332, 328

Boulanger, F., Abergel, A., Bernard, J.-P., et al. 1998a, in Star Formation with the Infrared Space Observatory, ed. J. Yun, \& R. Liseau (San Francisco: ASP), ASP Conf. Ser., 132, 15
Boulanger, F., Boissel, P., Cesarsky, D., \& Ryter, C. 1998b, A\&A, 339, 194

Carral, P., Hollenbach, D. J., Lord, S. D., et al. 1994, ApJ, 423, 223

Cesarsky, C. J., \& Sauvage, M. 1999, Ap\&SS, 269, 303

Cesarsky, D., Lequeux, J., Abergel, A., et al. 1996a, A\&A, 315, L305

Cesarsky, D., Lequeux, J., Abergel, A., et al. 1996b, A\&A, 315, L309

Cesarsky, C. J., Abergel, A., Agnèse, P., et al. 1996c, A\&A, 315, L32

Charmandaris, V., Laurent, O., Le Floc'h, E., et al. 2002, A\&A, 391, 429

Collison, P. M., Saikia, D. J., Pedlar, A., Axon, D. J., \& Unger, S. W. 1994, MNRAS, 268, 203

Coulais, A., \& Abergel, A. 2000, A\&AS, 141, 533

Crété, E., Giard, M., Joblin, C., et al. 1999, A\&A, 352, 277

Dahlem, M., Aalto, S., Klein, U., et al. 1990, A\&A, 240, 237

Dahlem, M., Ehle, M., \& Ryder, S. D., 2001, A\&A, 373, 485

Dale, D. A., Helou, G., Contursi, A., Silbermann, N. A., \& Kolhatkar, S. 2001, ApJ, 549, 215

Davidge, T. G., \& Pritchet, C. J. 1990, AJ, 100, 102

de Graauw, T., Haser, L. N., Beintema, D. A., et al. 1996, A\&A, 315, L49

Delaney, M. 1997, ISOCAM Interactive Analysis User's Manual, SAI-96-5226/Dc.

Désert, F. X., Boulanger, F., \& Puget, J. L. 1990, A\&A, 237, 215

Dietz, R. D., Smith, J., Hackwell, J. A., Gehrz, R. D., \& Grasdalen, G. L. 1986, AJ, 91, 758

Draine, B. T. 1989, in Proc. of the 22nd ESLAB Symp., Infrared Spectroscopy in Astronomy, ed. B. H. Kaldeich, ESA SP-290, 93

Draine, B. T., \& Li, A. 2001, ApJ, 551, 807

Dudley, C. C. 1999, MNRAS, 307,553

Dudley, C. C., \& Wynn-Williams, C. G. 1997, ApJ, 488, 720

Duley, W. W., \& Williams, D. A. 1991, MNRAS, 247, 647

Dwek, E., Arendt, R. G., Fixsen, D. J., et al. 1997, ApJ, 475, 565

Elbaz, D., Cesarsky, C. J., Chanial, P., et al. 2002, A\&A, 384, 848

Engelbracht, C. W., Rieke, M. J., Rieke, G. H., Kelly, D. M., \& Achtermann, J. M. 1998, ApJ, 505, 639

Förster Schreiber, N. M., Genzel, R., Lutz, D., Kunze, D., \& Sternberg, A. 2001, ApJ, 552, 544

Förster Schreiber, N. M., Genzel, R., Lutz, D., \& Sternberg, A. 2003, ApJ, submitted

Forbes, D. A., Boisson, C., \& Ward, M. J. 1992, MNRAS, 259, 293

Franx, M., Illingworth, G., Kelson, D., van Dokkum, P., \& Tran, K.-V. 1997, ApJ, 486, L75

Freedman, W. L., \& Madore, B. F. 1988, ApJ, 332, L63

Geballe, T. R. 1997, in From Stardust to Planetesimals, ed. Y. J. Pendleton, \& A. G. G. M. Tielens (San Francisco: ASP), ASP Conf. Ser., 122, 119

Genzel, R., Lutz, D., Sturm, E., et al. 1998, ApJ, 498, 579

Genzel, R., \& Cesarsky, C. 2000, ARA\&A, 38, 761

Giveon, U., Sternberg, A., Lutz, D., Feuchtgruber, H., \& Pauldrach, A. W. A. 2002, ApJ, 566, 880

Götz, M., McKeith, C. D., Downes, D., \& Greve, A. 1990, A\&A, 240, 52

Helou, G., Lu, N. Y., Werner, M. W., Malhotra, A., \& Silbermann, N. 2000, ApJ, 532, L21

Hudgins, D. M., \& Allamandola, L. J. 1999, ApJ, 516, L41

Joblin, C., Tielens, A. G. G. M., Geballe, T. R., \& Wooden, D. H. 1996, ApJ, 460, L119

Jones, A. P., \& d'Hendecourt, L. 2000, A\&A, 355, 1191

Junkes, N., Zinnecker, H., Hensler, G., Dahlem, M., \& Pietsch, W. 1995, A\&A, 294, 8

Kalas, P., \& Wynn-Williams, C. G. 1994, ApJ, 434, 546

Keto, E., Ball, R., Arens, J., et al. 1993, ApJ, 413, L23

Keto, E., Hora, J. L., Fazio, G. G., Hoffmann, W., \& Deutsch, L. 1999, ApJ, 518, 183 
Kessler, M. F., Steinz, J. A., Anderegg, M. E., et al. 1996, A\&A, 315, L27

Koribalski, B., Dahlem, M., Mebold, U., \& Brinks, E. 1993, A\&A, 268,14

Kotilainen, J. K., Forbes, D. A., Moorwood, A. F. M., et al. 1996, A\&A, 313, 771

Krabbe, A., Böker, T., \& Maiolino, R. 2001, ApJ, 557, 626

Krabbe, A., Sternberg, A., \& Genzel, R. 1994, ApJ, 425, 72

Larkin, J. E., Graham, J. R., Matthews, K., et al. 1994, ApJ, 420, 159

Laurent, O., Mirabel, I. F., Charmandaris, V., et al. 2000, A\&A, 359, 887

Le Floc'h, E., Charmandaris, V., Laurent, O., et al. 2002, A\&A, 391, 417

Léger, A., d'Hendecourt, L., \& Défourneau, D. 1989, A\&A, 216, 148

Li, A., \& Draine, B. T. 2002, ApJ, 572, 232

Lu, N. Y. 1998, ApJ, 498, L65

Lutz, D. 1999, in The Universe as seen by ISO, ed. P. Cox, \& M. F. Kessler, ESA SP-427, 623

Mattila, K., Lehtinen, K., \& Lemke, D. 1999, A\&A, 342, 643

Mirabel, I. F., Vigroux, L., Charmandaris, V., et al. 1998, A\&A, 333, L1

Normand, P., Rouan, D., Lacombe, F., \& Tiphène, D. 1995, A\&A, 297,311

Okumura, K. 2000, in ISO Beyond Point Sources: Studies of Extended Infrared Emission, ed. R. J. Laureijs, K. Leech, \& M. F. Kessler, ESA SP-455, 47

Onaka, T., Yamamura, I., Tanabé, T., Roellig, T. L., \& Yuen, L. 1996, PASJ, 48, L59

Pence, W. D. 1981, ApJ, 247, 473

Peng, R., Zhou, S., Whiteoak, J. B., Lo, K. Y., \& Sutton, E. C. 1996, ApJ, 470, 821

Pettini, M., Kellogg, M., Steidel, C., et al. 1998, ApJ, 508, 539

Piña, R. K., Jones, B., Puetter, R. C., \& Stein, W. A. 1992, ApJ, 401, L75

Puget, J.-L., Abergel, A., Bernard, J.-P., et al. 1996, A\&A, 308, L5

Puget, J.-L., \& Léger, A. 1989, ARA\&A, 27, 161

Reif, K., McBold, U., Goss, W. M., van Woerden, H., \& Siegman, B. 1982, A\&A, 50, 451

Rieke, G. H., Loken, K., Rieke, M. J., \& Tamblyn, P. 1993, ApJ, 412, 99

Rigopoulou, D., Spoon, H. W. W., Genzel, R., et al. 1999, AJ, 118, 2625

Ristorcelli, I., Giard, M., Meny, C., et al. 1994, A\&A, 286, L23

Roussel, H., Sauvage, M., Vigroux, L., et al. 2001a, A\&A, 372, 406

Roussel, H., Sauvage, M., Vigroux, L., \& Bosma, A. 2001b, A\&A, 372,427

Rowan-Robinson, M. 2001, ApJ, 549, 745
Saikia, D. J., Unger, S. W., Pedlar, A., et al. 1990, MNRAS, 245, 397 Sams, B. J. III, Genzel, R., Eckart, A., Tacconi-Garman, L., \& Hofmann, R. 1994, ApJ, 430, L33

Sandage, A. R., \& Tammann, G. A. 1987, A Revised Shapley-Ames Catalog of Bright Galaxies 2nd ed. (Carnegie Inst. Washington Publ.), vol. 635

Sanders, D. B., \& Mirabel, I. F. 1996, ARA\&A, 34, 749

Satyapal, S., Watson, D. M., Pipher, J. L., et al. 1995, ApJ, 448, 611

Schutte, W. A., Tielens, A. G. G. M., \& Allamandola, L. J. 1993, ApJ, 415,397

Seaquist, E. R., Carlstrom, J. E., Bryant, P. M., \& Bell, M. B. 1996, ApJ, 465, 691

Shen, J., \& Lo, K. Y. 1995, ApJ, 445, L99

Siebenmorgen, R., \& Krügel, E. 1992, A\&A, 259, 614

Siebenmorgen, R., Krügel, E., \& Laureijs, R. J. 2001, A\&A, 377, 735

Smail, I., Ivison, R. J., \& Blain, A. W. 1997, ApJ, 490, L5

Starck, J.-L., Abergel, A., Aussel, H., et al. 1999, A\&AS, 134, 135

Steidel, C. C., Giavalisco, M., Pettini, M., Dickinson, M., \& Adelberger, K. 1996, ApJ, 462, L17

Sturm, E., Lutz, D., Tran, D., et al. 2000, A\&A, 358, 481

Tacconi-Garman, L. E., Sternberg, A., \& Eckart, A. 1996, AJ, 112, 918

Telesco, C. M. 1988, ARA\&A, 26, 343

Telesco, C. M., Campins, H., Joy, M., Dietz, K., \& Decher, R. 1991, ApJ, 369, 135

Telesco, C. M., Dressel, L. L., \& Wolstencroft, R. D. 1993, ApJ, 414, 120

Telesco, C. M., \& Gezari, D. Y. 1992, ApJ, 395, 461

Thornley, M. D., Förster Schreiber, N. M., Lutz, D., et al. 2000, ApJ, 539,641

Tielens, A. G. G. M., Hony, S., van Kerckhoven, C., \& Peeters, E. 1999, in The Universe as seen by ISO, ed. P. Cox, \& M. F. Kessler, ESA SP-427, 579

Tokunaga, A. 1997, in Diffuse Infrared Radiation and the IRTS, ed. H. Okuda, T. Matsumoto, \& T. L. Roellig (San Francisco: ASP), ASP Conf. Ser., 124, 149

Tran, Q. D. 1998, Ph.D. Thesis, Université Paris XI

Tran, Q. D., Lutz, D., Genzel, R., et al. 2001, ApJ, 552, 527

Uchida, K. I., Sellgren, K., Werner, M. W., \& Houdashelt, M. L. 2000, ApJ, 530, 817

Ulvestad, J. S., \& Antonucci, R. R. J. 1997, ApJ, 488, 621

Verstraete, L., Puget, J.-L., Falgarone, E., et al. 1996, A\&A, 315, L337

Webster, B. L., \& Smith, M. G. 1983, MNRAS, 204, 743

Wills, K. A., Redman, M. P., Muxlow, T. W. B., \& Pedlar, A. 1999, MNRAS, 309, 395

Yun, M. S., Ho, P. T. P., \& Lo, K. Y. 1993, ApJ, 411, L17

Yun, M. S., Ho, P. T. P., \& Lo, K. Y. 1994, Nature, 372, 530 